\title{
O-Attack versus N-Attack: Electrophilic Halocyclization of Unsaturated Amides with Vinylic Halogen Substitution
}

Tianshun Hu, Kun Liu, Meihua Shen, Xinting Yuan, Yu Tang, and Chaozhong Li* Joint Laboratory of Green Synthetic Chemistry, Shanghai Institute of Organic Chemistry, Chinese Academy of Sciences, 354 Fenglin Road, Shanghai 200032, China

clig@mail.sioc.ac.cn

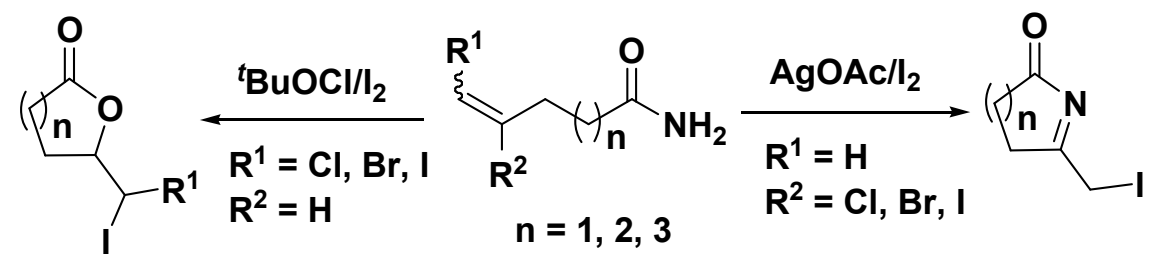

\section{Supporting Information}

\section{Table of Content}

1. Characterizations of Substrates 8a-b, 10a, 14.

2. Characterizations of Products $2,3,5,7,9,11,13,15,17 \mathbf{a}-\mathbf{b}, \mathbf{1 9}, \mathbf{2 1}, \mathbf{2 3 a}$-c. $\quad$ S3 - S 8

3. References for Known Compounds.

4. Computational Results on Halocyclization.

5. ${ }^{1} \mathrm{H}$ NMR Spectra of New Compounds. 
Characterization of substrates<smiles>C=C(Cl)Cc1ccccc1C(N)=O</smiles>

$8 a$

White solid. Mp 128-130 ${ }^{\circ} \mathrm{C} .{ }^{1} \mathrm{H}$ NMR $\left(300 \mathrm{MHz}, \mathrm{CDCl}_{3}\right) \delta 3.95(2 \mathrm{H}, \mathrm{s}), 5.15(1 \mathrm{H}, \mathrm{s}), 5.28$ $(1 \mathrm{H}, \mathrm{s}), 5.86(2 \mathrm{H}, \mathrm{br}), 7.30-7.36(2 \mathrm{H}, \mathrm{m}), 7.43(1 \mathrm{H}, \mathrm{t}, J=7.2 \mathrm{~Hz}), 7.51(1 \mathrm{H}, \mathrm{d}, J=7.5 \mathrm{~Hz})$. ${ }^{13} \mathrm{C}$ NMR $\left(75 \mathrm{MHz}, \mathrm{CDCl}_{3}\right) \delta 42.3,114.2,127.2,127.5,130.6,131.1,135.1,135.5,141.2$, 171.4. EIMS: $m / z$ (rel intensity) $196\left(\mathrm{M}^{+}+1,0.3\right), 160$ (100), 143 (15), 130 (11), 115 (58), 103 (4), 89 (10), 77 (8). Anal. calcd for $\mathrm{C}_{10} \mathrm{H}_{10} \mathrm{ClNO}$ : C, 61.39; H, 5.15; N, 7.16. Found: C, 61.48; H, 5.04; N, 7.00.<smiles>C=C(I)Cc1ccccc1C(N)=O</smiles>

8b

White solid. Mp 129-131 ${ }^{\circ} \mathrm{C} .{ }^{1} \mathrm{H}$ NMR $\left(300 \mathrm{MHz}, \mathrm{CDCl}_{3}\right) \delta 4.07$ (2H, s), $5.84(1 \mathrm{H}, \mathrm{s}), 5.92$ (2H, br), $6.04(1 \mathrm{H}, \mathrm{s}), 7.30-7.36(2 \mathrm{H}, \mathrm{m}), 7.44(1 \mathrm{H}, \mathrm{t}, J=7.5 \mathrm{~Hz}), 7.50(1 \mathrm{H}, \mathrm{d}, J=7.5 \mathrm{~Hz})$. ${ }^{13} \mathrm{C}$ NMR $\left(75 \mathrm{MHz}, \mathrm{CDCl}_{3}\right) \delta 48.6,109.2,127.3,127.5,127.6,130.6,131.1,135.4,136.2$, 171.4. EIMS: $m / z$ (rel intensity) $287\left(\mathrm{M}^{+}, 1\right), 184$ (2), 160 (98), 143 (15), 115 (100), 91 (22), 77 (16), 57 (20). Anal. calcd for $\mathrm{C}_{10} \mathrm{H}_{10} \mathrm{INO}$ : C, 41.83; H, 3.51; N, 4.88. Found: C, 41.58; H, $3.51 ; \mathrm{N}, 4.57$.<smiles>C=C(Cl)CCC(N)=O</smiles>

White Solid. Mp 70-72 ${ }^{\circ} \mathrm{C} .{ }^{1} \mathrm{H}$ NMR (300 MHz, $\left.\mathrm{CDCl}_{3}\right) \delta$ 2.49-2.54 (2H, m), 2.69-2.74 (2H, 
m), $5.22(1 \mathrm{H}, \mathrm{d}, J=1.2 \mathrm{~Hz}), 5.24(1 \mathrm{H}, \mathrm{d}, J=0.9 \mathrm{~Hz}), 5.63(1 \mathrm{H}, \mathrm{br}), 5.92(1 \mathrm{H}, \mathrm{br}) .{ }^{13} \mathrm{C} \mathrm{NMR}$ $\left(\mathrm{CDCl}_{3}\right) \delta 33.6,34.8,113.4,141.0,173.6$. EIMS: $m / z$ (rel. intensity) $134\left(\mathrm{M}^{+}+1,28\right), 117$ (1), 98 (100), 81 (5), 53 (38), 44 (62). Anal. calcd for $\mathrm{C}_{5} \mathrm{H}_{8} \mathrm{ClNO}$ : C, 44.96; H, 6.04; N, 10.49 . Found: C, 45.03; H, 6.16; N, 10.47 .<smiles>C=C(I)COCCC(N)=O</smiles>

White solid. Mp 38-40 ${ }^{\circ} \mathrm{C} .{ }^{1} \mathrm{H}$ NMR $\left(300 \mathrm{MHz} \mathrm{CDCl}_{3}\right) \delta 2.55(2 \mathrm{H}, \mathrm{t}, J=5.4 \mathrm{~Hz}), 3.70-3.74$ $(2 \mathrm{H}, \mathrm{m}), 4.09(2 \mathrm{H}, \mathrm{d}, J=1.2 \mathrm{~Hz}), 5.93-5.96(1 \mathrm{H}, \mathrm{m}), 5.53(1 \mathrm{H}, \mathrm{br}), 5.94(1 \mathrm{H}, \mathrm{d}, J=0.9 \mathrm{~Hz})$, $6.23(1 \mathrm{H}, \mathrm{br}), 6.38(1 \mathrm{H}, \mathrm{d}, J=1.5 \mathrm{~Hz}) .{ }^{13} \mathrm{C} \mathrm{NMR}\left(75 \mathrm{MHz}, \mathrm{CDCl}_{3}\right) \delta 36.4,65.9,78.5,106.5$, 127.0, 173.7. EIMS: $m / z$ (rel intensity) $256\left(\mathrm{M}^{+}+1,1\right), 167$ (12), 153 (1), 128 (12), 111 (1), 88 (8), 72 (100), 7 (8). HRMS calcd for $\mathrm{C}_{6} \mathrm{H}_{11} \mathrm{INO}_{2}(\mathrm{M}+\mathrm{H})$ : 255.9834. Found: 255.9835. Anal. calcd for $\mathrm{C}_{6} \mathrm{H}_{10} \mathrm{INO}_{2}$ : C, 28.25; H, 3.95; N, 5.49. Found: C, 27.96; H, 3.93; N, 5.44.

\section{Characterization of products}<smiles>O=C1CCCC(CI)=N1</smiles>

2

Yellowish oil. ${ }^{1} \mathrm{H}$ NMR (300 MHz, $\left.\mathrm{CDCl}_{3}\right) \delta$ 1.94-2.04 (2H, m), 2.45 (2H, t, J=6.9 Hz), 2.95 $(2 \mathrm{H}, \mathrm{t}, J=6.8 \mathrm{~Hz}), 3.84(2 \mathrm{H}, \mathrm{d}, J=0.9 \mathrm{~Hz}) .{ }^{13} \mathrm{C} \mathrm{NMR}\left(75 \mathrm{MHz}, \mathrm{CDCl}_{3}\right) \delta 5.6,16.1,19.6$, 36.8, 119.0, 201.4. EIMS: $m / z$ (rel intensity) $237\left(\mathrm{M}^{+}, 12\right), 209$ (4), 195 (3), 169 (17), 141 (11), 110 (16), 96 (100), 68 (48). Anal. calcd for $\mathrm{C}_{6} \mathrm{H}_{8} \mathrm{INO}$ : C, 30.40; H, 3.40; N, 5.91. Found: C, $30.42 ; \mathrm{H}, 3.52 ; \mathrm{N}, 5.83$. 
<smiles>O=C1CCCC(CCl)=N1</smiles>

3

Colorless oil. ${ }^{1} \mathrm{H}$ NMR (300 MHz, $\mathrm{CDCl}_{3}$ ) $\delta$ 1.95-2.04 (2H, m), 2.44-2.49 (2H, m), 2.80-2.85 $(2 \mathrm{H}, \mathrm{m}), 4.10-4.12(2 \mathrm{H}, \mathrm{m}) .{ }^{13} \mathrm{C} \mathrm{NMR}\left(75 \mathrm{MHz}, \mathrm{CDCl}_{3}\right) \delta 16.3,19.1,37.4,47.9,118.9,201.2$. EIMS: $m / z$ (rel intensity) $146\left(\mathrm{M}^{+}+1,5\right), 125$ (1), 105 (1), 96 (100), 83 (3), 77 (11), 68 (68), 55 (25). Anal. calcd for $\mathrm{C}_{6} \mathrm{H}_{8} \mathrm{ClNO}$ : $\mathrm{C}, 49.50 ; \mathrm{H}, 5.54 ; \mathrm{N}, 9.62$. Found: $\mathrm{C}, 49.61 ; \mathrm{H}, 5.74 ; \mathrm{N}$, 9.48 .

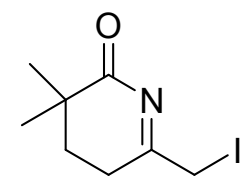

5

White solid. ${ }^{1} \mathrm{H}$ NMR (300 MHz, $\left.\mathrm{CDCl}_{3}\right) \delta 1.38(6 \mathrm{H}, \mathrm{s}), 1.87-1.93(2 \mathrm{H}, \mathrm{m}), 2.94-2.99(2 \mathrm{H}$, m), $3.87(2 \mathrm{H}, \mathrm{s}) .{ }^{13} \mathrm{C} \mathrm{NMR}\left(75 \mathrm{MHz}, \mathrm{CDCl}_{3}\right) \delta$ 5.3, 26.6, 31.9, 34.8, 35.2, 124.2, 201.4. EIMS: $m / z$ (rel intensity) $266\left(\mathrm{M}^{+}+1,19\right), 239$ (15), 221 (2), 169 (17), 141 (10), 124 (100), 96 (37), 69 (39). HRMS calcd for $\mathrm{C}_{8} \mathrm{H}_{13} \mathrm{INO}(\mathrm{M}+\mathrm{H}): 266.0042$. Found: 266.0028.<smiles>[Z]C1(C)CCC(=O)N=C1CI</smiles>

Yellow oil. ${ }^{1} \mathrm{H}$ NMR (300 MHz, $\left.\mathrm{CDCl}_{3}\right) \delta 1.22(6 \mathrm{H}, \mathrm{s}), 1.87-1.92(2 \mathrm{H}, \mathrm{m}), 2.22-2.27(2 \mathrm{H}, \mathrm{m})$, $3.92(2 \mathrm{H}, \mathrm{s}) .{ }^{13} \mathrm{C} \mathrm{NMR}\left(75 \mathrm{MHz}, \mathrm{CDCl}_{3}\right) \delta 1.3,13.1,25.0,35.2,47.3,119.5,206.2$. EIMS: m/z (rel intensity) $166\left(\mathrm{M}^{+}+1,3\right), 237$ (1), 169 (10), 141 (5), 127 (3), 96 (100), 69 (36), 55 (45). HRMS calcd for $\mathrm{C}_{8} \mathrm{H}_{13} \mathrm{NO}(\mathrm{M}+\mathrm{H}-\mathrm{I})$ : 139.1004. Found: 139.0097.<smiles>O=C1N=C(CI)Cc2ccccc21</smiles> 
Red oil. ${ }^{1} \mathrm{H}$ NMR (300 MHz, $\left.\mathrm{CDCl}_{3}\right) \delta 3.98(2 \mathrm{H}, \mathrm{s}), 4.29(2 \mathrm{H}, \mathrm{s}), 7.36-7.44(2 \mathrm{H}, \mathrm{m})$, 7.57-7.69 (2H, m). ${ }^{13} \mathrm{C} \mathrm{NMR}\left(75 \mathrm{MHz}, \mathrm{CDCl}_{3}\right) \delta$ 5.2, 44.0, 113.5, 117.6, 128.0, 130.9, 132.8, 133.0, 137.4, 198.3. EIMS: $m / z$ (rel intensity) $286\left(\mathrm{M}^{+}+1,3\right), 257$ (6), 243 (11), 169 (18), 141 (6), 116 (100), 89 (26), 77 (5). HRMS calcd for $\mathrm{C}_{10} \mathrm{H}_{8} \mathrm{INO}(\mathrm{M}): 284.9651$. Found: 284.9664.

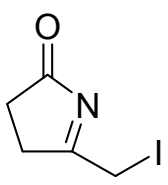

11

Yellow oil. ${ }^{1} \mathrm{H}$ NMR $\left(300 \mathrm{MHz}, \mathrm{CDCl}_{3}\right) \delta 2.64(2 \mathrm{H}, \mathrm{t}, J=6.9 \mathrm{~Hz}), 3.15(2 \mathrm{H}, \mathrm{t}, J=6.9 \mathrm{~Hz})$, $3.87(2 \mathrm{H}, \mathrm{s}) .{ }^{13} \mathrm{C} \mathrm{NMR}\left(75 \mathrm{MHz}, \mathrm{CDCl}_{3}\right) \delta 4.1,11.9,34.3,118.5,199.0$. EIMS: $m / z$ (rel intensity) $223\left(\mathrm{M}^{+}, 53\right), 195$ (26), 169 (28), 141 (24), 127 (26), 82 (100), 54 (63), 42 (72). HRMS calcd for $\mathrm{C}_{5} \mathrm{H}_{6} \mathrm{INO}(\mathrm{M}): 222.9494$. Found: 222.9505.

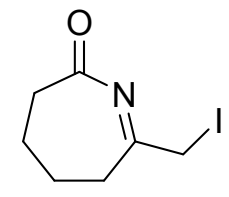

13

Yellow oil. ${ }^{1} \mathrm{H}$ NMR (300 MHz, $\left.\mathrm{CDCl}_{3}\right) \delta$ 1.67-1.82 (4H, m), $2.39(2 \mathrm{H}, \mathrm{t}, J=7.2 \mathrm{~Hz}), 2.81$ $(2 \mathrm{H}, \mathrm{t}, J=7.2 \mathrm{~Hz}), 3.81(2 \mathrm{H}, \mathrm{s}) .{ }^{13} \mathrm{C} \mathrm{NMR}\left(75 \mathrm{MHz}, \mathrm{CDCl}_{3}\right) \delta 5.6,17.1,22.9,24.6,37.9$, 119.3, 202.0. EIMS: $m / z$ (rel intensity) $252\left(\mathrm{M}^{+}+1,6\right), 234$ (1), 169 (11), 124 (20), 110 (87), 82 (100), 55 (91). HRMS calcd for $\mathrm{C}_{7} \mathrm{H}_{10} \mathrm{INO}(\mathrm{M}): 250.9807$. Found: 250.9832.

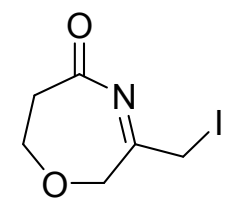

15

Yellow oil. ${ }^{1} \mathrm{H}$ NMR (300 MHz, $\left.\mathrm{CDCl}_{3}\right) \delta 2.71(2 \mathrm{H}, \mathrm{t}, J=6.3 \mathrm{~Hz}), 3.80(2 \mathrm{H}, \mathrm{t}, J=6.0 \mathrm{~Hz})$, $3.92(2 \mathrm{H}, \mathrm{s}), 4.41(2 \mathrm{H}, \mathrm{s}),{ }^{13} \mathrm{C} \mathrm{NMR}\left(75 \mathrm{MHz}, \mathrm{CDCl}_{3}\right) \delta 0.9,18.9,66.3,72.9,117.3,200.5$. EIMS $m / z$ (rel intensity) $253\left(\mathrm{M}^{+}, 1\right), 183$ (1), 169 (6), 141 (5), 127 (6), 84 (58), 54 (100). Anal. calcd for $\mathrm{C}_{6} \mathrm{H}_{8} \mathrm{INO}_{2}$ : C, 28.48; H, 3.19; N, 5.54. Found: C, 28.65; H, 3.08; N, 5.42. 
<smiles>O=C1CCCC(C(Cl)I)O1</smiles>

Yellow oil. Two stereoisomers in $\sim 2.3: 1$ ratio. ${ }^{1} \mathrm{H}$ NMR $\left(300 \mathrm{MHz}, \mathrm{CDCl}_{3}\right) \delta 1.77-2.06(2 \mathrm{H}$, m), 2.29-2.34 (1H, m), 2.40-2.56 (2H, m), 2.63-2.69 $(1 \mathrm{H}, \mathrm{m}), 4.00-4.04$ and 4.56-4.59 $(1 \mathrm{H}$, m), $5.87(1 \mathrm{H}, \mathrm{d}, J=1.2 \mathrm{~Hz}) .{ }^{13} \mathrm{C}$ NMR $\left(75 \mathrm{MHz}, \mathrm{CDCl}_{3}\right) \delta$ 17.8/18.0, 23.9/25.8, 29.4/29.6, 29.7/32.2, 82.1/83.3, 169.4. EIMS: $m / z$ (rel intensity) $274\left(\mathrm{M}^{+}, 1\right), 239$ (2), 169 (1), 147 (15), 119 (50), 99 (38), 77 (56), 55 (100). HRMS calcd for $\mathrm{C}_{6} \mathrm{H}_{8} \mathrm{ClIO}_{2}$ (M): 273.9258. Found: 273.9257.<smiles>O=C1CCCC(C(I)I)O1</smiles>

Yellowish oil. ${ }^{1} \mathrm{H}$ NMR $\left(300 \mathrm{MHz}, \mathrm{CDCl}_{3}\right) \delta$ 1.62-1.71 (1H, m), 1.83-1.96 (2H, m), 2.36-2.48 $(2 \mathrm{H}, \mathrm{m}), 2.53-2.62(1 \mathrm{H}, \mathrm{m}), 3.85(1 \mathrm{H}, \mathrm{dt}, J=10.5,3.6 \mathrm{~Hz}), 5.21(1 \mathrm{H}, \mathrm{d}, J=3.6 \mathrm{~Hz}) .{ }^{13} \mathrm{C} \mathrm{NMR}$ $\left(75 \mathrm{MHz}, \mathrm{CDCl}_{3}\right) \delta-23.4,17.8,27.5,29.3,82.7,176.8$. EIMS: $m / z$ (rel intensity) $366\left(\mathrm{M}^{+}\right.$, 21), 239 (32), 211 (100), 169 (37), 141 (12), 127 (9), 99 (16), 55 (31). HRMS calcd for $\mathrm{C}_{6} \mathrm{H}_{8} \mathrm{I}_{2} \mathrm{O}_{2}(\mathrm{M}): 365.8614$. Found: 365.8617.

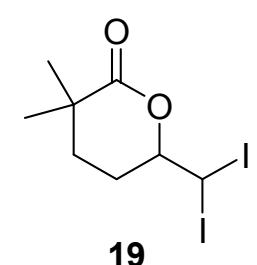

Yellow oil. ${ }^{1} \mathrm{H}$ NMR (300 MHz, $\left.\mathrm{CDCl}_{3}\right) \delta 1.32(3 \mathrm{H}, \mathrm{s}), 1.36(3 \mathrm{H}, \mathrm{s}), 1.75-1.95(4 \mathrm{H}, \mathrm{m})$, 2.32-2.38 (1H, m), 3.83-3.89 (1H, m), $5.28(1 \mathrm{H}, \mathrm{d}, J=3.6 \mathrm{~Hz}) \cdot{ }^{13} \mathrm{C} \mathrm{NMR}\left(75 \mathrm{MHz}, \mathrm{CDCl}_{3}\right) \delta$ $-21.6,1.0,25.1,27.2 / 27.5,33.1 / 37.5,83.3,175.5$. EIMS: $m / z$ (rel intensity) $394\left(\mathrm{M}^{+}, 22\right), 349$ (1), 293 (1), 267 (23), 239 (86), 169 (56), 127 (44), 41 (100). HRMS calcd for $\mathrm{C}_{8} \mathrm{H}_{13} \mathrm{I}_{2} \mathrm{O}_{2}$ (M+H): 394.9005. Found: 394.8986. 
<smiles>CC1(C)CCC(=O)OC1C(Cl)I</smiles>

21

Yellowish oil. Two stereoisomers in $\sim 5: 1$ ratio. ${ }^{1} \mathrm{H}$ NMR $\left(300 \mathrm{MHz}, \mathrm{CDCl}_{3}\right) \delta 1.11(3 \mathrm{H}, \mathrm{s})$, $1.22(3 \mathrm{H}, \mathrm{s}), 1.65-1.79(2 \mathrm{H}, \mathrm{m}), 2.60-2.68(2 \mathrm{H}, \mathrm{m}), 4.11$ and $4.62(1 \mathrm{H}, 2 \mathrm{~m}), 5.94$ and 6.21 (1H, 2br). ${ }^{13} \mathrm{C}$ NMR (75 MHz, $\left.\mathrm{CDCl}_{3}\right) \delta$ 21.0/22.5, 23.1/25.0, 27.1/27.2, 27.8/29.7, 33.2/33.5, 34.9, 89.8/91.5, 169.2. EIMS: $m / z$ (rel intensity) $175\left(\mathrm{M}^{+}-\mathrm{I}, 17\right), 147$ (10), 127 (12), 97 (31), 83 (12), 69 (93), 55 (85), 40 (100). HRMS calcd for $\mathrm{C}_{8} \mathrm{H}_{12} \mathrm{ClIO}_{2}$ (M): 301.9571. Found: 301.9573.

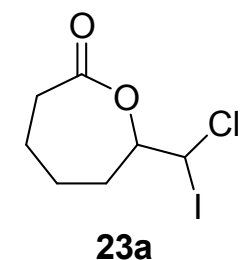

Yellow oil. Two stereoisomers in $\sim 13: 1$ ratio. ${ }^{1} \mathrm{H}$ NMR $\left(300 \mathrm{MHz}, \mathrm{CDCl}_{3}\right) \delta 1.59-1.69(2 \mathrm{H}$, m), 1.73-1.90 (1H, m), 1.99-2.09 (2H, m), 2.29-2.44 (1H, m), 2.54-2.63 (1H, m), 2.74-2.81 $(1 \mathrm{H}, \mathrm{m}), 4.38(1 \mathrm{H}, \mathrm{d}, J=8.7 \mathrm{~Hz}), 5.82(1 \mathrm{H}, \mathrm{d}, J=1.5 \mathrm{~Hz}) .{ }^{13} \mathrm{C} \mathrm{NMR}\left(75 \mathrm{MHz}, \mathrm{CDCl}_{3}\right) \delta 22.8$, 27.5, 30.2, 31.6, 34.8, 83.8, 172.6. EIMS: $m / z$ (rel intensity) $288\left(\mathrm{M}^{+}, 1\right), 253$ (3), 161 (22), 127 (9), 113 (18), 97 (20), 79 (43), 55 (100). HRMS calcd for $\mathrm{C}_{7} \mathrm{H}_{10} \mathrm{ClIO}_{2}$ (M): 287.9414. Found: 287.9423.

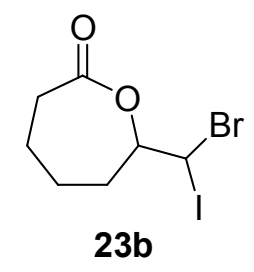

Red oil. Two stereoisomers in $\sim 2.4: 1$ ratio. ${ }^{1} \mathrm{H}$ NMR (300 MHz, $\left.\mathrm{CDCl}_{3}\right) \delta 1.61-1.75(1 \mathrm{H}, \mathrm{m})$, 1.82-2.12 (4H, m), 2.31-2.36 (1H, m), 2.55-2.65 (1H, m), 2.76-2.83 (1H, m), 4.10-4.17 and 4.35-4.39 (1H, 2m), 5.61-5.63 (1H, m). $\left.{ }^{13} \mathrm{C} \mathrm{NMR} \mathrm{(75} \mathrm{MHz,} \mathrm{CDCl}_{3}\right) \delta 12.5,22.8,27.4$, 32.9/33.5, 34.9, 83.5, 172.5. EIMS: $m / z$ (rel intensity) $333\left(\mathrm{M}^{+}+1,2\right), 253$ (27), 207 (19), 167 
(6), 141 (5), 127 (20), 97 (51), 41 (100). Anal. calcd for $\mathrm{C}_{7} \mathrm{H}_{10} \mathrm{BrIO}_{2}$ : C, 25.25; H, 3.03. Found: C, 25.50; H, 3.09.

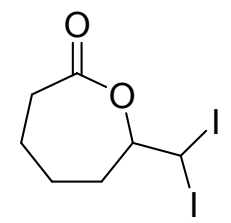

23c

Yellowish oil. ${ }^{1} \mathrm{H}$ NMR (300 MHz, $\left.\mathrm{CDCl}_{3}\right) \delta$ 1.57-1.67 (2H, m), 1.82-2.06 (3H, m), 2.25-2.30 $(1 \mathrm{H}, \mathrm{m}), 2.54-2.62(1 \mathrm{H}, \mathrm{m}), 2.76-2.82(1 \mathrm{H}, \mathrm{m}), 3.99-4.02(1 \mathrm{H}, \mathrm{m}), 5.22-5.24(1 \mathrm{H}, \mathrm{m}) .{ }^{13} \mathrm{C}$ NMR (75 MHz, $\left.\mathrm{CDCl}_{3}\right) \delta-24.5,22.8,27.2,34.8,35.1,83.2,172.4$. EIMS: $m / z$ (rel intensity) $380\left(\mathrm{M}^{+}, 4\right), 252$ (48), 225 (4), 169 (38), 125 (37), 97 (63), 79 (78), 41 (100). HRMS calcd for $\mathrm{C}_{7} \mathrm{H}_{10} \mathrm{I}_{2} \mathrm{O}_{2}(\mathrm{M}): 379.8770$. Found: 379.8787 .

\section{References for known compounds}

\begin{tabular}{|c|c|}
\hline Compound & Literature \\
\hline $\begin{array}{c}1 \mathrm{a}, 1 \mathrm{~b}, 1 \mathrm{c}, 4,6,12 \mathrm{a}, 12 \mathrm{~b}, \\
12 \mathrm{c}, 16 \mathrm{a}, 16 \mathrm{~b}, 18,20,22 \mathrm{a}, \\
22 \mathrm{~b}, 22 \mathrm{c}\end{array}$ & Hu, T.; Shen, M.; Chen, Q.; Li, C. Org. Lett. 2006, 8, 2647. \\
\hline $10 b$ & Hu, T.; Li, C. Org. Lett. 2005, 7, 2035. \\
\hline
\end{tabular}




\section{Computational (UB3LYP/6-31G*) Results}

\section{TS-1}

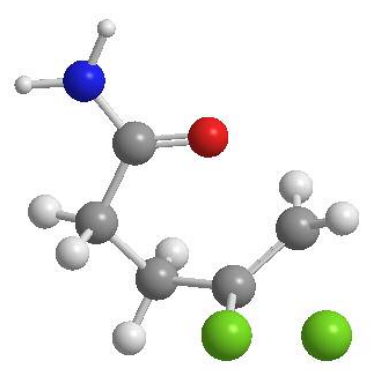

\#p b31yp/6-31g* opt=(ts,z-matrix,noeigentest,nrscale,calcfc) $\quad$ scfcyc $=200 \quad$ optcyc $=500$ freq $=$ noraman $\operatorname{iop}(1 / 8=5)$

$\mathrm{X}=\mathrm{H}, \mathrm{Y}=\mathrm{H}$; TSOA $\quad \mathrm{HF}=-1245.4174605$

11

C

$\begin{array}{lll}\mathrm{O} & 1 & 2.58782923\end{array}$

$\begin{array}{lllll}\mathrm{C} & 1 & 1.50588556 & 2 & 84.87120437\end{array}$

$\begin{array}{lllllll}\mathrm{H} & 3 & 1.09308868 & 1 & 110.04685820 & 2 & -155.82934866\end{array}$

$\begin{array}{llllllr}\mathrm{H} & 3 & 1.09418828 & 1 & 108.72151381 & 2 & 85.78983771\end{array}$

$\begin{array}{lllllll}\mathrm{C} & 3 & 1.54949076 & 1 & 110.02595409 & 2 & -35.38108697\end{array}$

$\begin{array}{lllllll}\mathrm{H} & 6 & 1.09472598 & 3 & 108.09629791 & 1 & 172.51032402\end{array}$

$\begin{array}{lllllll}\mathrm{H} & 6 & 1.09696943 & 3 & 109.78855231 & 1 & -71.36801988\end{array}$

$\begin{array}{lllllll}\mathrm{C} & 2 & 1.24537008 & 1 & 91.98509029 & 3 & 23.29077631\end{array}$

$\begin{array}{lllllll}\mathrm{N} & 9 & 1.33910214 & 2 & 121.33465400 & 1 & 178.16490012\end{array}$

$\begin{array}{lllllll}\mathrm{H} & 10 & 1.01339629 & 9 & 118.97675964 & 2 & -2.16728178\end{array}$

$\begin{array}{lllllll}\mathrm{H} & 10 & 1.01083694 & 9 & 122.79431432 & 2 & -177.97374534\end{array}$

$\begin{array}{lllllll}\mathrm{C} & 1 & 1.44410815 & 3 & 119.53027203 & 6 & -87.40577517\end{array}$

$\begin{array}{lllllll}\mathrm{H} & 13 & 1.08127729 & 1 & 119.95689864 & 3 & -12.39569288\end{array}$

$\begin{array}{lllllll}\mathrm{H} & 13 & 1.08474151 & 1 & 120.56679134 & 3 & 157.17901651\end{array}$

$\begin{array}{lllllll}\mathrm{Cl} & 13 & 2.05451251 & 1 & 69.49500582 & 3 & -104.53643898\end{array}$

$\begin{array}{llllllr}\mathrm{Cl} & 1 & 1.72120382 & 13 & 118.29248316 & 2 & -89.33994428\end{array}$

\section{TS-2}




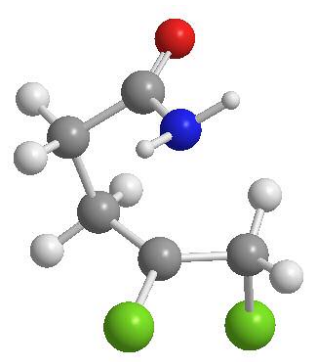

\#p b3lyp/6-31g* opt=(ts,z-matrix,noeigentest,nrscale,calcfc) $\quad$ scfcyc $=200 \quad$ optcyc $=500$ freq $=$ noraman $\operatorname{iop}(1 / 8=5)$

$\mathrm{X}=\mathrm{Cl}, \mathrm{Y}=\mathrm{H} ; \mathrm{TSNA} \quad \mathrm{HF}=-1245.4080166$

$\begin{array}{ccccccc}11 & & & & & \\ \mathrm{~N} & & & & & & \\ \mathrm{C} & 1 & 2.70998339 & & & & \\ \mathrm{C} & 2 & 1.48674508 & 1 & 89.16170335 & & \\ \mathrm{H} & 3 & 1.09482916 & 2 & 110.08056005 & 1 & -137.71050314 \\ \mathrm{H} & 3 & 1.09354973 & 2 & 108.85022258 & 1 & 102.27041352 \\ \mathrm{C} & 3 & 1.57239040 & 2 & 110.80460107 & 1 & -17.27526894 \\ \mathrm{H} & 6 & 1.09112798 & 3 & 107.75556930 & 2 & 169.37472542 \\ \mathrm{H} & 6 & 1.09446088 & 3 & 109.92103566 & 2 & -71.59013845 \\ \mathrm{C} & 1 & 1.39982080 & 6 & 35.28512507 & 3 & 109.18732215 \\ \mathrm{O} & 9 & 1.21042839 & 1 & 122.58236964 & 6 & -167.72133629 \\ \mathrm{H} & 1 & 1.01877626 & 9 & 113.59914561 & 10 & 12.03519708 \\ \mathrm{H} & 1 & 1.01655179 & 9 & 117.76677950 & 10 & 147.09605458 \\ \mathrm{C} & 2 & 1.48755043 & 1 & 88.50747435 & 9 & 104.01453331 \\ \mathrm{H} & 13 & 1.09092261 & 2 & 111.99990117 & 1 & -54.54011728 \\ \mathrm{H} & 13 & 1.08831045 & 2 & 114.13682583 & 1 & 74.60708783 \\ \mathrm{Cl} & 13 & 1.81623208 & 2 & 99.56539397 & 1 & -169.16219675 \\ \mathrm{Cl} & 2 & 1.67887155 & 1 & 99.31169372 & 9 & -137.48045943\end{array}$

The transition state for O-attack in the halocyclization of 4-pentenamide based on the model

24:

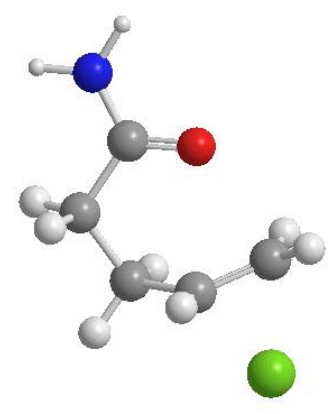


\#p b31yp/6-31g* opt=(ts,z-matrix,noeigentest,nrscale,calcfc) $\quad$ scfcyc $=200 \quad$ optcyc $=500$ freq $=$ noraman $\operatorname{iop}(1 / 8=5)$

$\mathrm{X}=\mathrm{H}, \mathrm{Y}=\mathrm{H} ; \mathrm{TSOA} \quad \mathrm{HF}=-785.8337455$

$\begin{array}{lcccccc}11 & & & & & \\ \mathrm{C} & & & & & \\ \mathrm{O} & 1 & 2.53779925 & & & & \\ \mathrm{C} & 1 & 1.49935043 & 2 & 84.96905480 & & \\ \mathrm{H} & 3 & 1.09409968 & 1 & 109.94648044 & 2 & -156.13387457 \\ \mathrm{H} & 3 & 1.09447249 & 1 & 110.27149206 & 2 & 84.61957095 \\ \mathrm{C} & 3 & 1.54987413 & 1 & 109.21804084 & 2 & -37.04719552 \\ \mathrm{H} & 6 & 1.09470546 & 3 & 108.54685040 & 1 & 175.52687796 \\ \mathrm{H} & 6 & 1.09821180 & 3 & 110.13162340 & 1 & -68.06204112 \\ \mathrm{C} & 2 & 1.24271080 & 1 & 93.09899505 & 3 & 23.53395841 \\ \mathrm{~N} & 9 & 1.34038425 & 2 & 121.98114511 & 1 & 179.80725208 \\ \mathrm{H} & 10 & 1.01316373 & 9 & 118.98100400 & 2 & -1.80415352 \\ \mathrm{H} & 10 & 1.01056156 & 9 & 122.82934189 & 2 & -178.52273206 \\ \mathrm{C} & 1 & 1.43874172 & 3 & 122.09897475 & 6 & -95.70911509 \\ \mathrm{H} & 13 & 1.08387858 & 1 & 120.10297982 & 3 & -10.54679329 \\ \mathrm{H} & 13 & 1.08501672 & 1 & 120.55667027 & 3 & 155.57484337 \\ \mathrm{Cl} & 1 & 2.00008995 & 13 & 69.26627753 & 2 & 174.24417362 \\ \mathrm{H} & 1 & 1.08823447 & 13 & 116.53149682 & 2 & -90.18777876\end{array}$

The transition state for $\mathrm{N}$-attack in the halocyclization of 4-pentenamide based on the model 24:

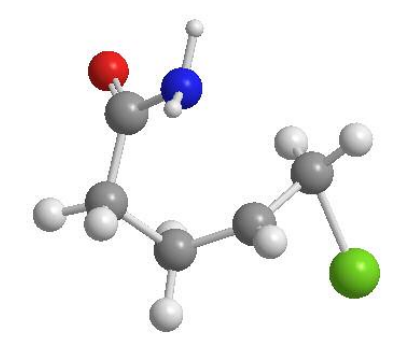

\#p b31yp/6-31g* opt=(ts,z-matrix,noeigentest,nrscale,calcfc) $\quad$ scfcyc $=200 \quad$ optcyc $=500$ freq $=$ noraman $\operatorname{iop}(1 / 8=5)$

$\mathrm{X}=\mathrm{H}, \mathrm{Y}=\mathrm{H} ; \mathrm{TSNA} \quad \mathrm{HF}=-785.8118578$

11 


$\begin{array}{ccccccc}\mathrm{N} & & & & & & \\ \mathrm{C} & 1 & 2.77075076 & & & & \\ \mathrm{C} & 2 & 1.48446986 & 1 & 89.91209279 & & \\ \mathrm{H} & 3 & 1.09525947 & 2 & 110.05739945 & 1 & -136.01417511 \\ \mathrm{H} & 3 & 1.09321839 & 2 & 110.97211855 & 1 & 102.67280673 \\ \mathrm{C} & 3 & 1.56921927 & 2 & 108.51346059 & 1 & -17.42494993 \\ \mathrm{H} & 6 & 1.09164380 & 3 & 107.58854198 & 2 & 170.84884811 \\ \mathrm{H} & 6 & 1.09489262 & 3 & 110.25262545 & 2 & -70.43970264 \\ \mathrm{C} & 1 & 1.39811671 & 6 & 35.19926614 & 3 & 105.29844865 \\ \mathrm{O} & 9 & 1.21087637 & 1 & 122.80125283 & 6 & -168.19788090 \\ \mathrm{H} & 1 & 1.01827147 & 9 & 113.53349448 & 10 & 13.16280548 \\ \mathrm{H} & 1 & 1.01611221 & 9 & 117.62481733 & 10 & 147.86935809 \\ \mathrm{C} & 2 & 1.46038193 & 1 & 90.20086616 & 9 & 105.19559612 \\ \mathrm{H} & 13 & 1.08856802 & 2 & 117.58807428 & 1 & -77.92007616 \\ \mathrm{H} & 13 & 1.08694445 & 2 & 117.96783967 & 1 & 67.54034924 \\ \mathrm{Cl} & 13 & 1.85144129 & 2 & 80.97376220 & 1 & 174.53453736 \\ \mathrm{H} & 2 & 1.08849311 & 1 & 87.51121520 & 9 & -138.09284299\end{array}$

\section{TS-3}

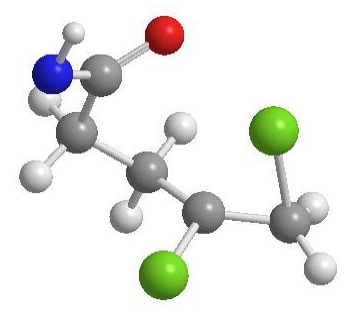

\#p $\quad$ b3lyp/6-31 g* opt=(ts,z-matrix,noeigentest,nrscale,calcfc) $\quad$ scfcyc $=200 \quad$ optcyc $=500$ freq=noraman $\operatorname{iop}(1 / 8=5)$

$\mathrm{X}=\mathrm{Cl}, \mathrm{Y}=\mathrm{H} ; \mathrm{TSOA} \quad \mathrm{HF}=-1244.9390914$

01

$\mathrm{O}$

$\begin{array}{lll}\mathrm{C} & 1 & 3.18396020\end{array}$

$\begin{array}{lllll}\mathrm{N} & 1 & 2.34605339 & 2 & 90.81919031\end{array}$

$\begin{array}{lllllll}\mathrm{C} & 2 & 1.48710601 & 1 & 110.19873702 & 3 & -153.44080217\end{array}$

$\begin{array}{lllllll}\mathrm{H} & 4 & 1.08628932 & 2 & 117.92056712 & 1 & 129.20848498\end{array}$

$\begin{array}{llllllr}\mathrm{C} & 2 & 1.50186771 & 1 & 64.29912260 & 3 & 86.20936211\end{array}$

$\begin{array}{lllllll}\mathrm{H} & 6 & 1.10068941 & 2 & 106.54600952 & 1 & -174.77229618\end{array}$

$\begin{array}{llllllr}\mathrm{H} & 6 & 1.09451950 & 2 & 107.36947343 & 1 & 69.44776643\end{array}$

$\begin{array}{lllllll}\text { C } & 6 & 1.54704122 & 2 & 114.07794246 & 1 & -52.26346994\end{array}$ 


$\begin{array}{lcccccc}\mathrm{H} & 9 & 1.09255316 & 6 & 110.67709193 & 2 & -70.61631188 \\ \mathrm{H} & 9 & 1.09733585 & 6 & 105.92952305 & 2 & 173.39840921 \\ \mathrm{C} & 1 & 1.29198237 & 3 & 25.21143622 & 9 & -0.86824876 \\ \mathrm{H} & 4 & 1.08761033 & 2 & 117.50019801 & 1 & -83.42198354 \\ \mathrm{H} & 3 & 1.02803798 & 1 & 81.93984536 & 12 & -179.65837075 \\ \mathrm{Cl} & 2 & 1.73454729 & 1 & 110.61411225 & 12 & -42.89644526 \\ \mathrm{Cl} & 4 & 1.87162768 & 2 & 71.39209538 & 1 & 20.43438405\end{array}$

\section{TS-4}

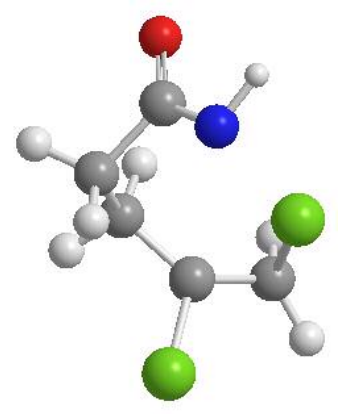

\#p $\quad$ b3lyp/6-31g* opt=(ts,z-matrix,noeigentest,nrscale,calcfc) $\quad$ scfcyc $=200 \quad$ optcyc $=500$ freq=noraman $\operatorname{iop}(1 / 8=5)$

$\mathrm{X}=\mathrm{Cl}, \mathrm{Y}=\mathrm{H}$; TSNA $\quad \mathrm{HF}=-1244.943537$

01

$\mathrm{O}$

$\begin{array}{lrrrrrr}\mathrm{C} & 1 & 3.93591099 & & & & \\ \mathrm{~N} & 1 & 2.34809666 & 2 & 62.91124065 & & \\ \mathrm{C} & 2 & 1.49364351 & 1 & 103.07901868 & 3 & -101.34718601 \\ \mathrm{H} & 4 & 1.08640483 & 2 & 117.45052423 & 1 & 160.29255453 \\ \mathrm{C} & 2 & 1.48893230 & 1 & 43.67689577 & 3 & 129.75417091 \\ \mathrm{H} & 6 & 1.09944353 & 2 & 105.67867319 & 1 & -177.82698901 \\ \mathrm{H} & 6 & 1.09602957 & 2 & 109.83859112 & 1 & 64.45938076 \\ \mathrm{C} & 6 & 1.55881710 & 2 & 114.32562143 & 1 & -56.77260829 \\ \mathrm{H} & 9 & 1.09320929 & 6 & 110.78637367 & 2 & -45.46189474 \\ \mathrm{H} & 9 & 1.09364085 & 6 & 106.37724610 & 2 & -164.00561302 \\ \mathrm{C} & 1 & 1.24907642 & 3 & 25.87814573 & 9 & 2.85068102 \\ \mathrm{H} & 4 & 1.08826931 & 2 & 116.55512988 & 1 & -55.76782137 \\ \mathrm{H} & 3 & 1.02912249 & 1 & 81.69867364 & 12 & -176.03474474 \\ \mathrm{Cl} & 4 & 1.85440384 & 2 & 74.48904501 & 1 & 51.06826261 \\ \mathrm{Cl} & 2 & 1.73336929 & 1 & 133.57265606 & 12 & 19.75102259\end{array}$




\section{TS-5}

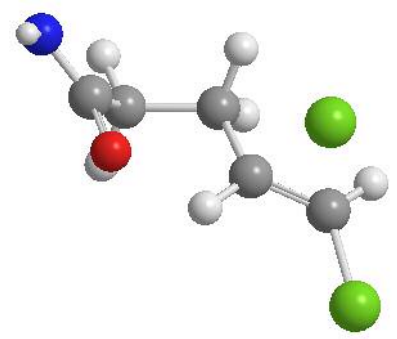

\#p $\quad$ b3lyp/6-31 g* opt=(ts,z-matrix,noeigentest,nrscale,calcfc) $\quad$ scfcyc $=200 \quad$ optcyc $=500$ freq=noraman $\operatorname{iop}(1 / 8=5)$

$\mathrm{X}=\mathrm{H}, \mathrm{Y}=\mathrm{Cl}$; TSOA $\quad \mathrm{HF}=-1244.9482753$

$\begin{array}{lrrrrrr}01 & & & & & \\ \mathrm{O} & & & & & & \\ \mathrm{C} & 1 & 2.70923940 & & & & \\ \mathrm{~N} & 1 & 2.31320444 & 2 & 113.27180266 & & \\ \mathrm{C} & 2 & 1.40754743 & 1 & 154.87919025 & 3 & 161.29733138 \\ \mathrm{H} & 2 & 1.09416657 & 1 & 37.78116461 & 3 & -159.29999946 \\ \mathrm{C} & 2 & 1.50656386 & 1 & 83.44890194 & 3 & -10.22165200 \\ \mathrm{H} & 6 & 1.09964880 & 2 & 108.94305368 & 1 & -152.66143276 \\ \mathrm{H} & 6 & 1.09734819 & 2 & 109.66761896 & 1 & 91.39185607 \\ \mathrm{C} & 6 & 1.53863810 & 2 & 111.47185453 & 1 & -30.15779565 \\ \mathrm{H} & 9 & 1.09779097 & 6 & 109.59934910 & 2 & -61.97192716 \\ \mathrm{H} & 9 & 1.09237780 & 6 & 109.43039400 & 2 & 179.34222741 \\ \mathrm{C} & 1 & 1.27496522 & 3 & 27.32571880 & 9 & 1.18035921 \\ \mathrm{H} & 4 & 1.08621168 & 2 & 121.59623416 & 1 & -161.11712100 \\ \mathrm{H} & 3 & 1.02822861 & 1 & 81.09563907 & 12 & -174.51645499 \\ \mathrm{Cl} & 2 & 2.25956820 & 1 & 93.64484673 & 12 & 111.16555001 \\ \mathrm{Cl} & 4 & 1.70621874 & 2 & 123.11558745 & 1 & 32.14769881\end{array}$

\section{TS-6}




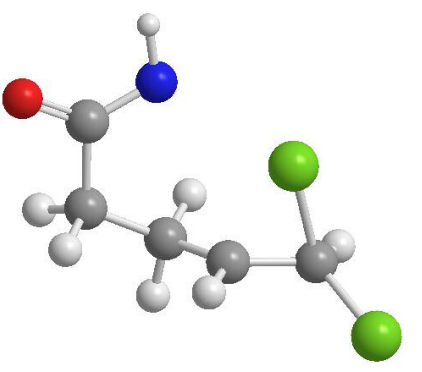

\#p b3lyp/6-31g* opt=(ts,z-matrix,noeigentest,nrscale,calcfc) $\quad$ scfcyc $=200 \quad$ optcyc $=500$ freq=noraman $\operatorname{iop}(1 / 8=5)$

$\mathrm{X}=\mathrm{H}, \mathrm{Y}=\mathrm{Cl}$; TSNA $\quad \mathrm{HF}=-1244.9415719$

$\begin{array}{lrrrrrr}01 & & & & & \\ \mathrm{O} & & & & & & \\ \mathrm{C} & 1 & 4.41297101 & & & & \\ \mathrm{~N} & 1 & 2.33351614 & 2 & 44.90595866 & & \\ \mathrm{C} & 2 & 1.47614959 & 1 & 141.60377061 & 3 & 12.06857524 \\ \mathrm{H} & 2 & 1.08908060 & 1 & 85.33237732 & 3 & 137.03887088 \\ \mathrm{C} & 2 & 1.47482957 & 1 & 53.65886399 & 3 & -92.59635425 \\ \mathrm{H} & 6 & 1.10642207 & 2 & 103.36619171 & 1 & -139.54018081 \\ \mathrm{H} & 6 & 1.09534479 & 2 & 110.80637052 & 1 & 104.95185026 \\ \mathrm{C} & 6 & 1.54413996 & 2 & 114.90205976 & 1 & -21.82056866 \\ \mathrm{H} & 9 & 1.09796667 & 6 & 110.18015238 & 2 & -48.03834298 \\ \mathrm{H} & 9 & 1.09551136 & 6 & 108.12015335 & 2 & -163.30613885 \\ \mathrm{C} & 1 & 1.24806204 & 3 & 25.90519166 & 9 & 1.16733104 \\ \mathrm{H} & 4 & 1.08679433 & 2 & 118.05529945 & 1 & -79.18316860 \\ \mathrm{H} & 3 & 1.02594929 & 1 & 83.13429228 & 12 & 178.92359219 \\ \mathrm{Cl} & 4 & 1.86082639 & 2 & 74.44051307 & 1 & 23.34511556 \\ \mathrm{Cl} & 4 & 1.75694835 & 2 & 119.36025062 & 1 & 139.50720709\end{array}$

The transition state for O-attack in the halocyclization of 4-pentenamide based on the model 27:

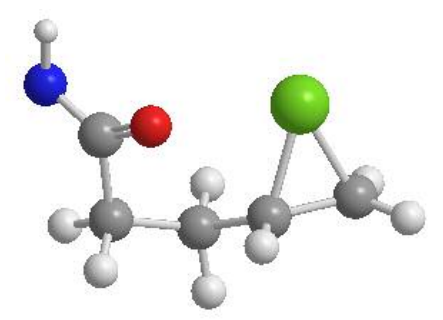

\#p $\quad$ b31yp/6-31g* opt=(ts,z-matrix,noeigentest,nrscale,calcfc $) \quad$ scfcyc $=200 \quad$ optcyc $=500$ 


\begin{tabular}{|c|c|c|c|c|c|c|}
\hline \multicolumn{4}{|c|}{$\mathrm{X}=\mathrm{H}, \mathrm{Y}=\mathrm{H} ; \mathrm{TSOA}$} & & & \\
\hline \multicolumn{7}{|l|}{01} \\
\hline \multicolumn{7}{|l|}{$\mathrm{O}$} \\
\hline $\mathrm{C}$ & 1 & 2.74117795 & & & & \\
\hline $\mathrm{N}$ & 1 & 2.35686992 & 2 & 109.19411753 & & \\
\hline $\mathrm{C}$ & 2 & 1.47879001 & 1 & 132.11970924 & 3 & 106.47800168 \\
\hline $\mathrm{H}$ & 2 & 1.08651985 & 1 & 73.42923049 & 3 & -141.46724354 \\
\hline $\mathrm{H}$ & 4 & 1.08637513 & 2 & 119.97684589 & 1 & 98.89366243 \\
\hline $\mathrm{C}$ & 2 & 1.49968718 & 1 & 82.24924940 & 3 & -20.69526623 \\
\hline $\mathrm{H}$ & 7 & 1.10490082 & 2 & 103.42582838 & 1 & -145.46578963 \\
\hline $\mathrm{H}$ & 7 & 1.09710403 & 2 & 109.59162920 & 1 & 101.10747989 \\
\hline $\mathrm{C}$ & 7 & 1.53704873 & 2 & 115.12761378 & 1 & -24.92126564 \\
\hline $\mathrm{H}$ & 10 & 1.09826234 & 7 & 108.88197907 & 2 & -64.47394747 \\
\hline $\mathrm{H}$ & 10 & 1.09290097 & 7 & 108.82436765 & 2 & 177.47372784 \\
\hline $\mathrm{C}$ & 1 & 1.28864397 & 3 & 24.67153867 & 10 & -1.16153764 \\
\hline $\mathrm{H}$ & 4 & 1.08658383 & 2 & 118.92819719 & 1 & -105.07737842 \\
\hline $\mathrm{H}$ & 3 & 1.02852790 & 1 & 82.31032348 & 13 & 178.60246741 \\
\hline $\mathrm{Cl}$ & 2 & 1.90594844 & 1 & 64.91005066 & 13 & 117.81424047 \\
\hline
\end{tabular}

The transition state for $\mathrm{N}$-attack in the halocyclization of 4-pentenamide based on the model 27:

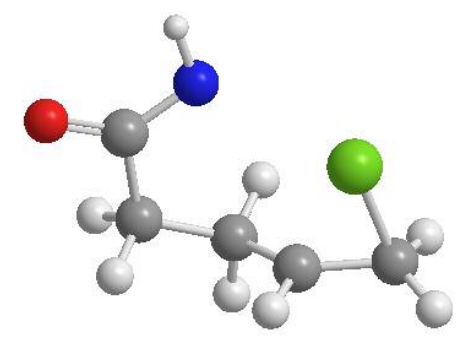

\#p b31yp/6-31g* opt=(ts,z-matrix,noeigentest,nrscale,calcfc) $\quad$ scfcyc $=200 \quad$ optcyc $=500$ freq=noraman $\operatorname{iop}(1 / 8=5)$

$\mathrm{X}=\mathrm{H}, \mathrm{Y}=\mathrm{H} ; \mathrm{TSNA} \quad \mathrm{HF}=-785.3528769$

01

$\mathrm{O}$

C $\quad 1 \quad 4.43096101$

$\begin{array}{lllll}\mathrm{N} & 1 & 2.33648216 & 2 & 44.33541424\end{array}$ 


$\begin{array}{lrrrrrr}\mathrm{C} & 2 & 1.47664830 & 1 & 145.25643039 & 3 & 6.81866321 \\ \mathrm{H} & 2 & 1.08930867 & 1 & 82.52728995 & 3 & 131.61651021 \\ \mathrm{H} & 4 & 1.08742435 & 2 & 118.83736176 & 1 & 130.02325393 \\ \mathrm{C} & 2 & 1.47836130 & 1 & 53.27905560 & 3 & -96.26697210 \\ \mathrm{H} & 7 & 1.10645908 & 2 & 103.44685144 & 1 & -139.39489674 \\ \mathrm{H} & 7 & 1.09589436 & 2 & 110.45530079 & 1 & 105.68824617 \\ \mathrm{C} & 7 & 1.54162570 & 2 & 115.09158011 & 1 & -21.18180595 \\ \mathrm{H} & 10 & 1.09874250 & 7 & 109.91356036 & 2 & -49.84707223 \\ \mathrm{H} & 10 & 1.09564900 & 7 & 108.03885190 & 2 & -164.65505024 \\ \mathrm{C} & 1 & 1.24917526 & 3 & 25.79275758 & 10 & 0.91985595 \\ \mathrm{H} & 4 & 1.08726362 & 2 & 118.10476050 & 1 & -81.51256805 \\ \mathrm{H} & 3 & 1.02610928 & 1 & 82.59992038 & 13 & 178.23978754 \\ \mathrm{Cl} & 4 & 1.85858506 & 2 & 73.06220966 & 1 & 22.20634283\end{array}$


${ }^{1}$ H NMR Spectra of New Compounds:

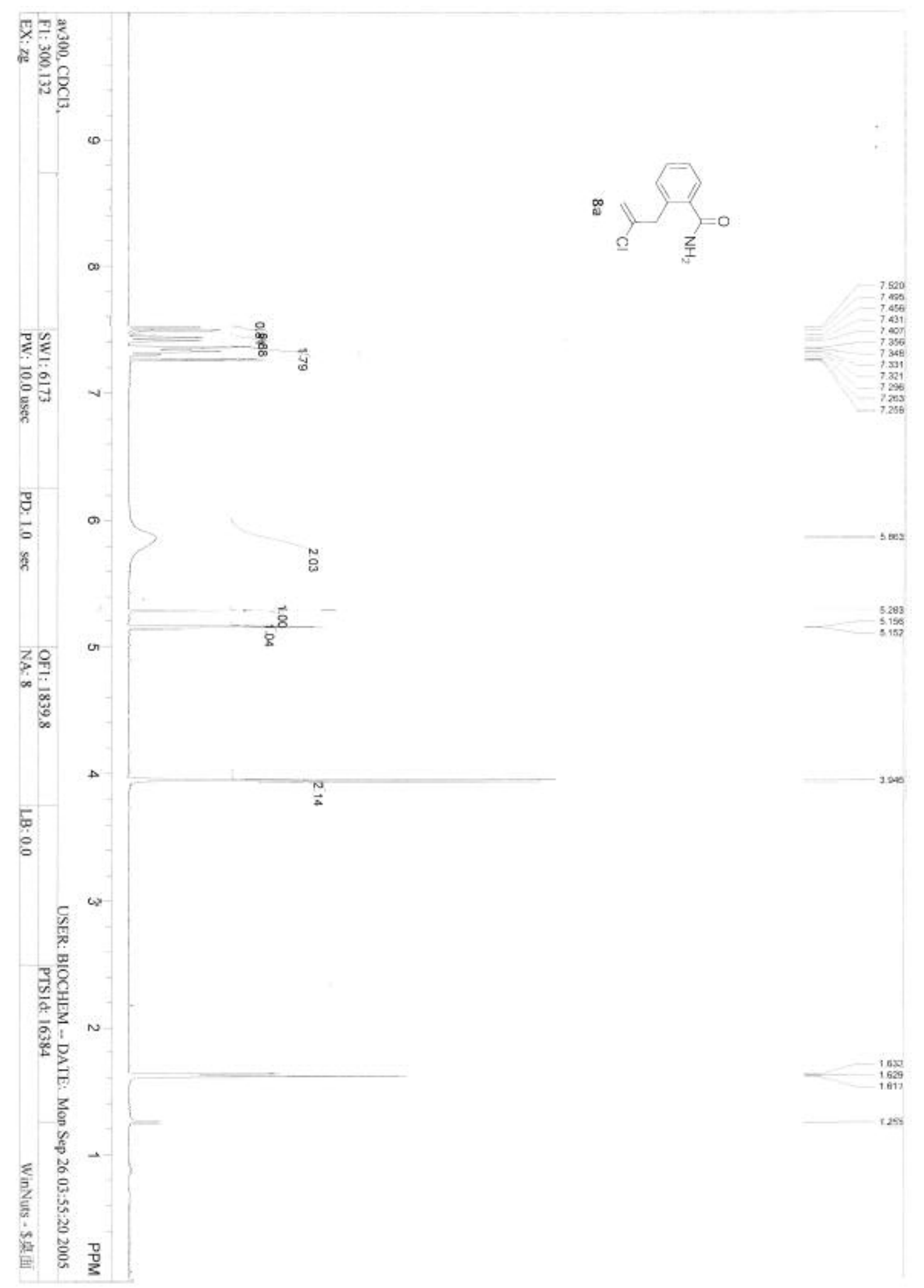




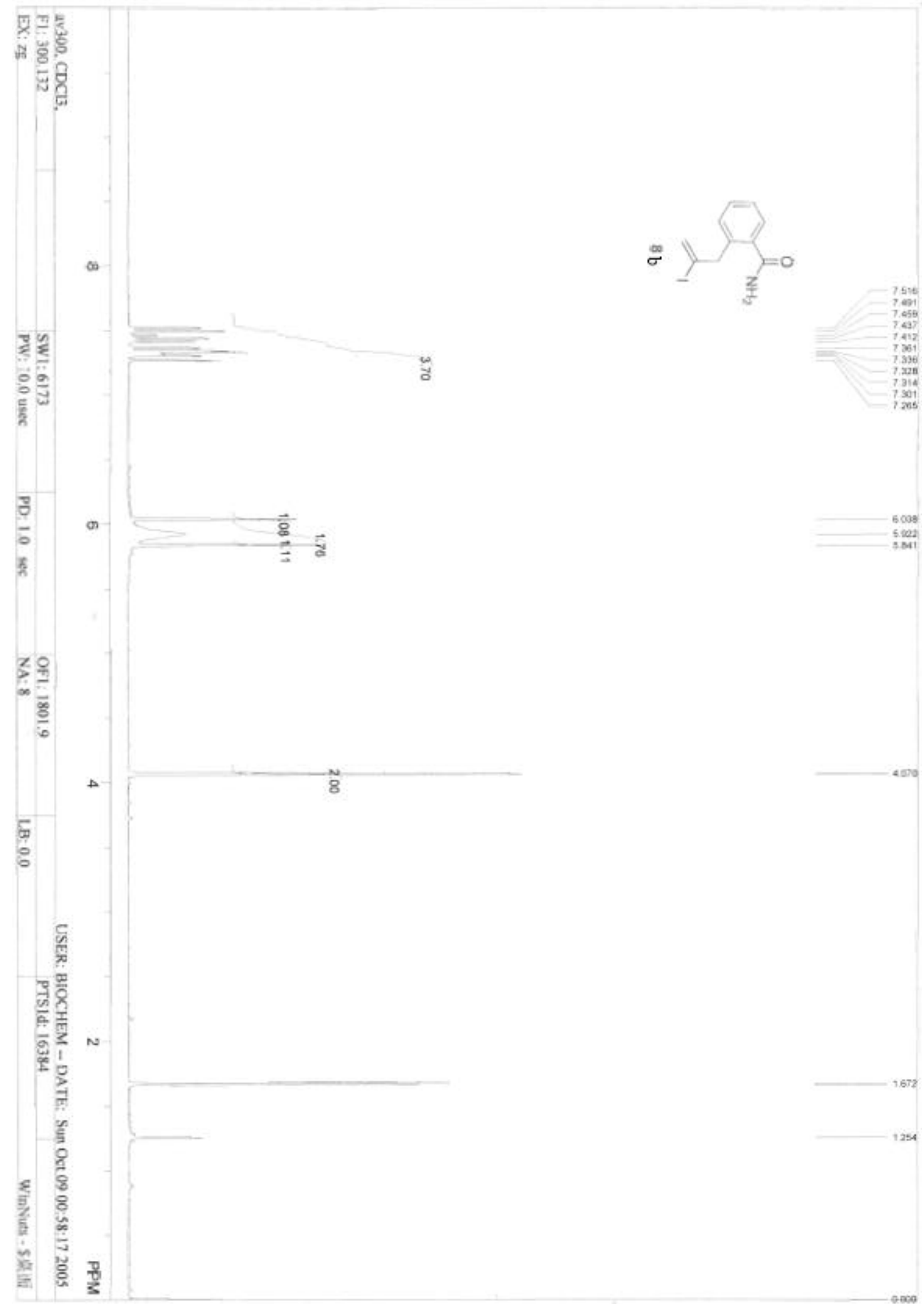




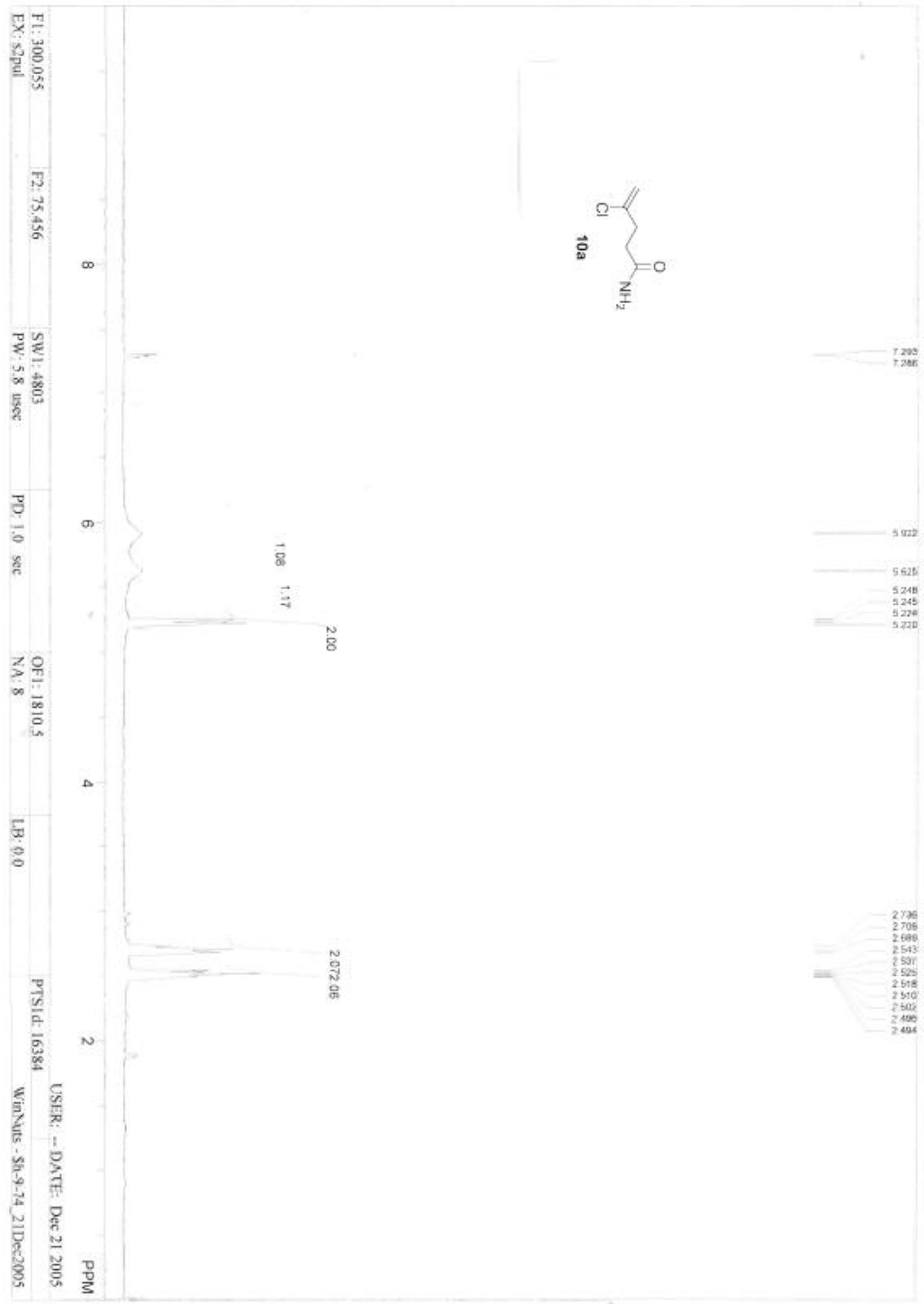




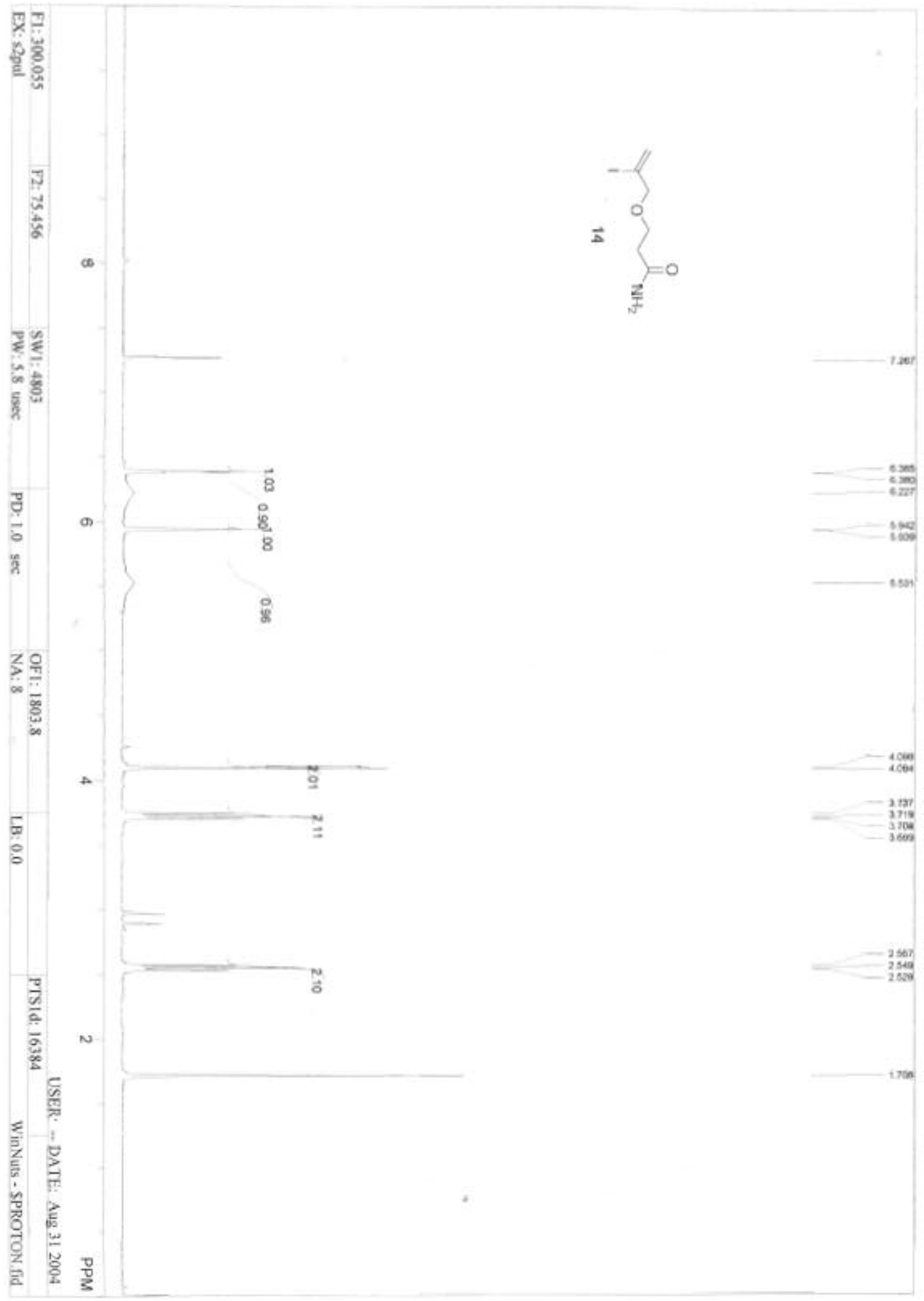




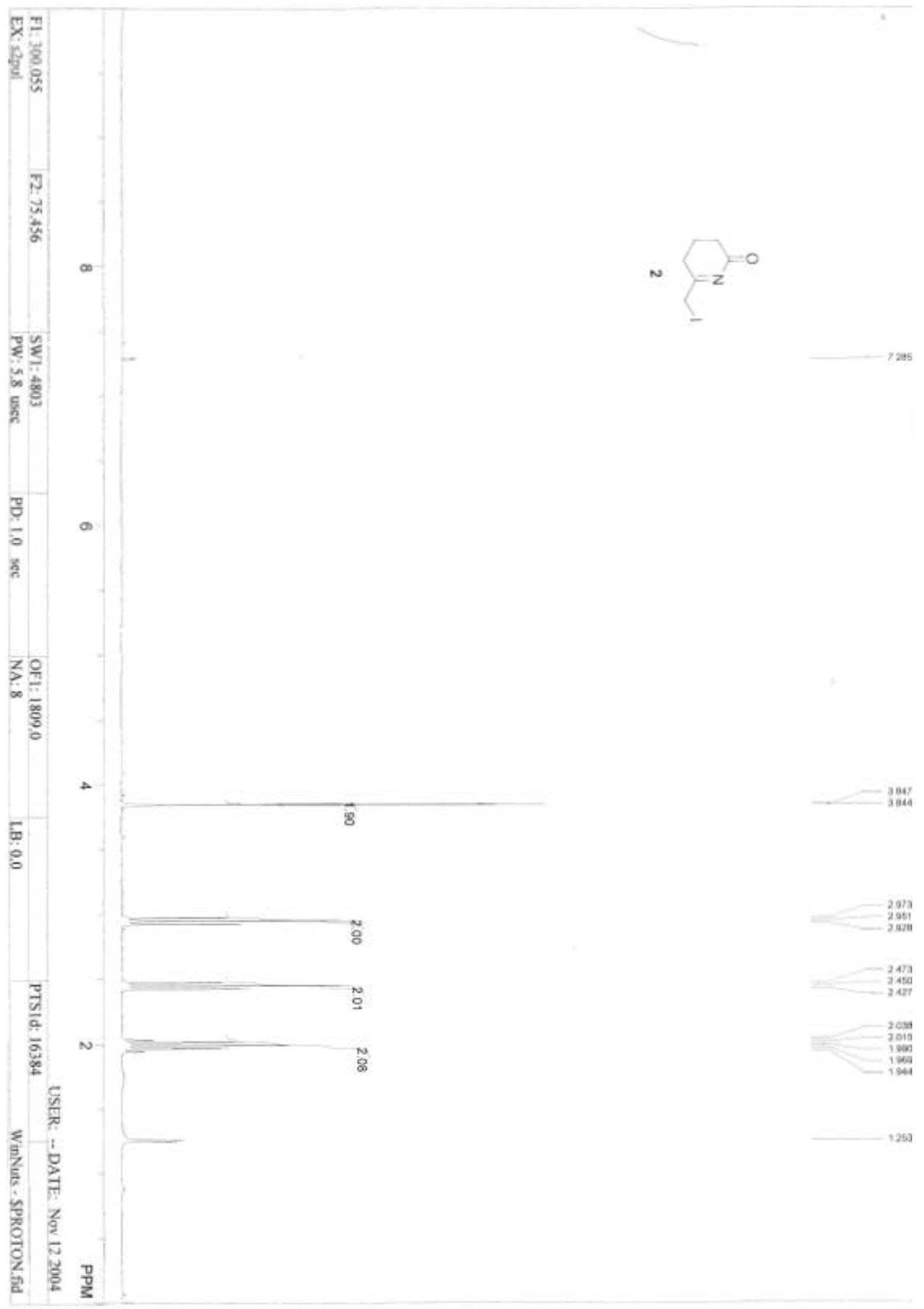




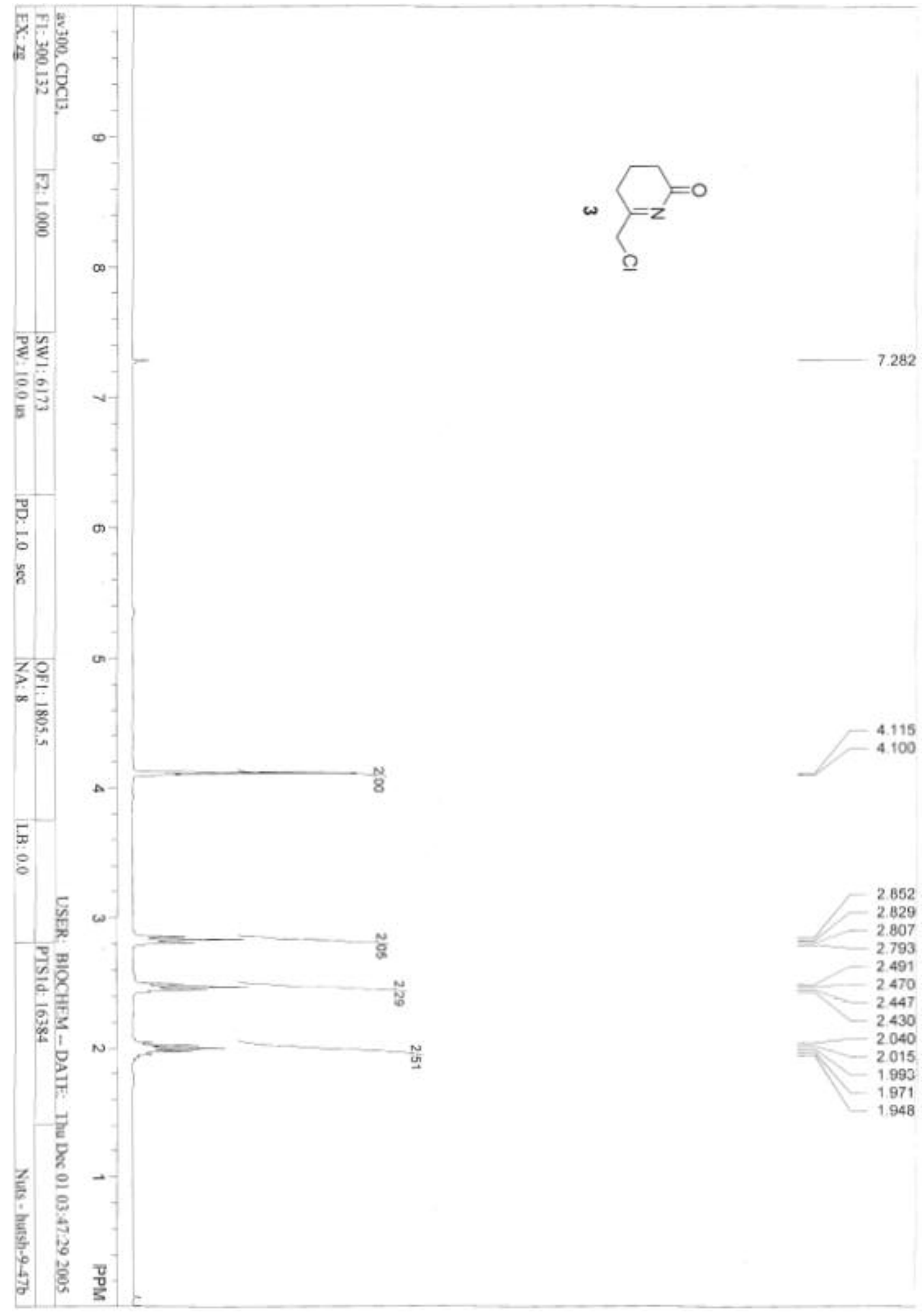




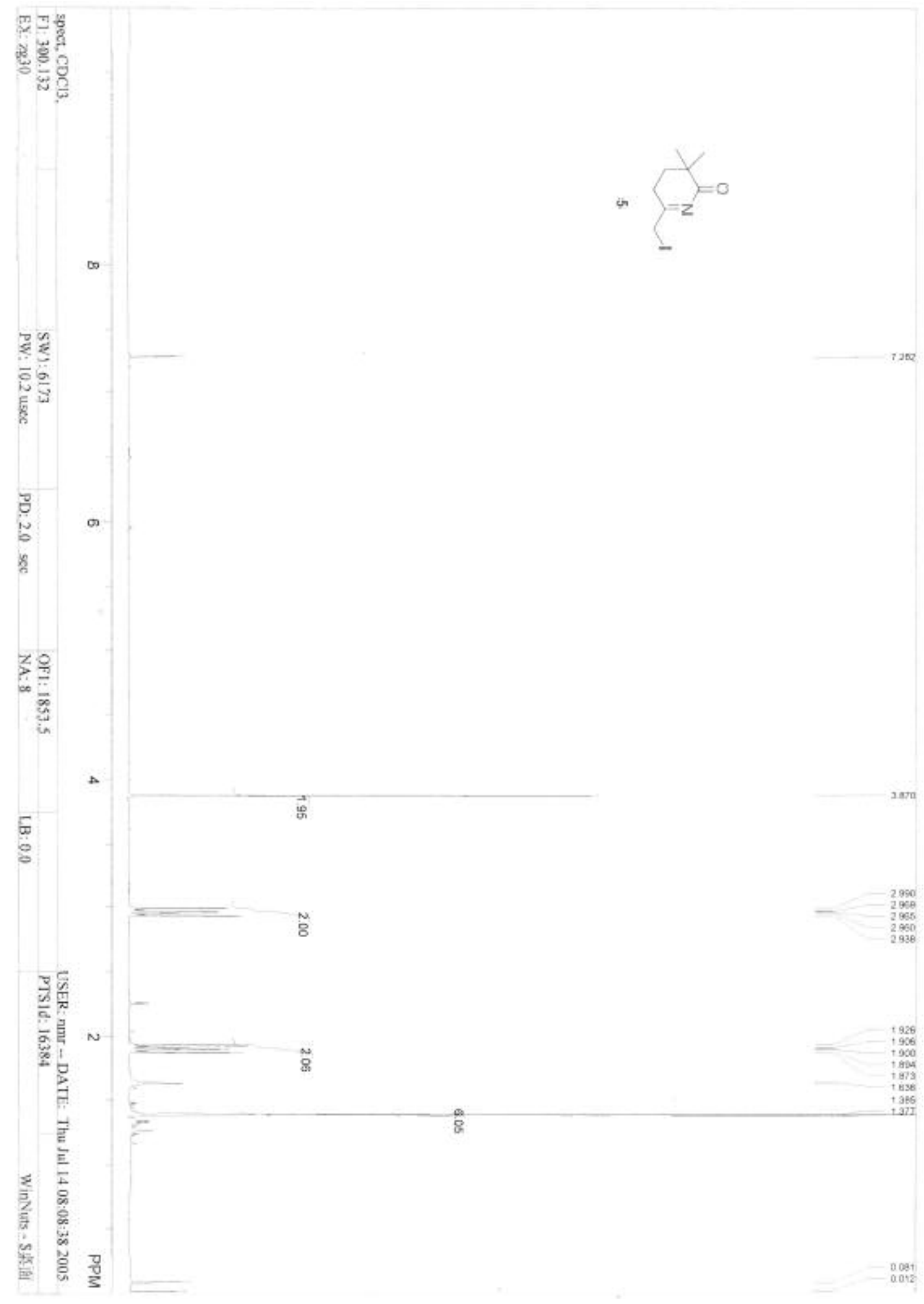




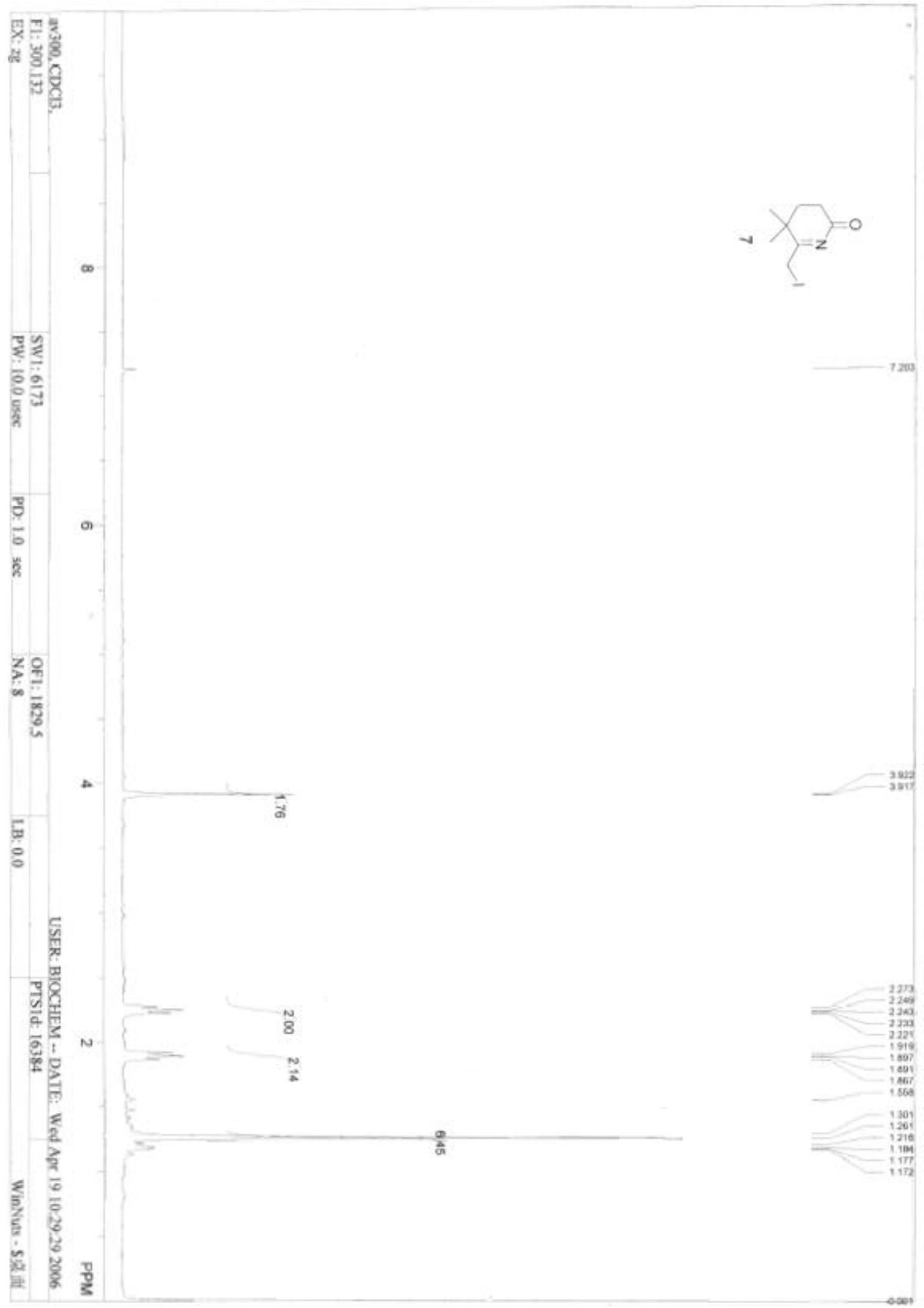




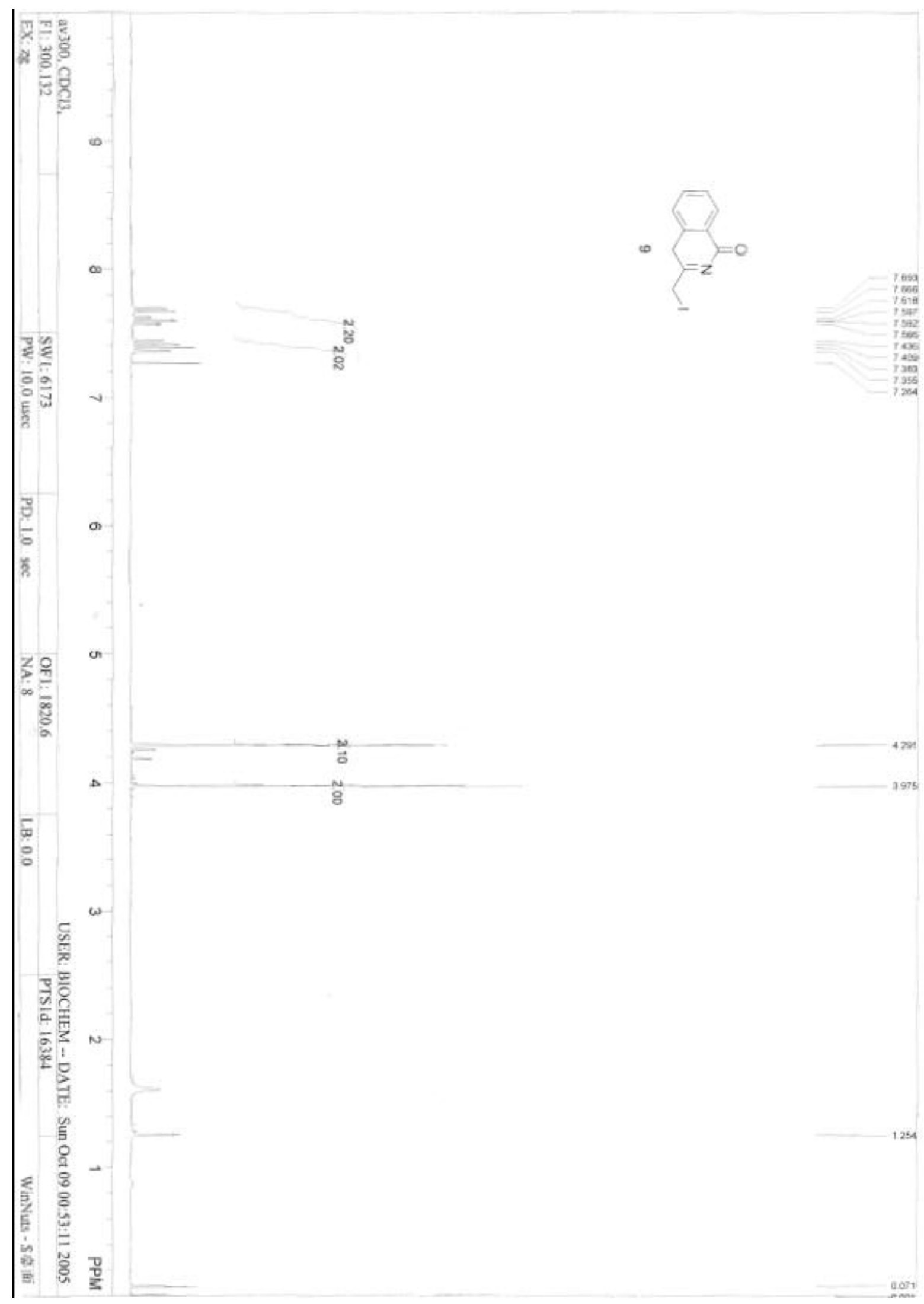




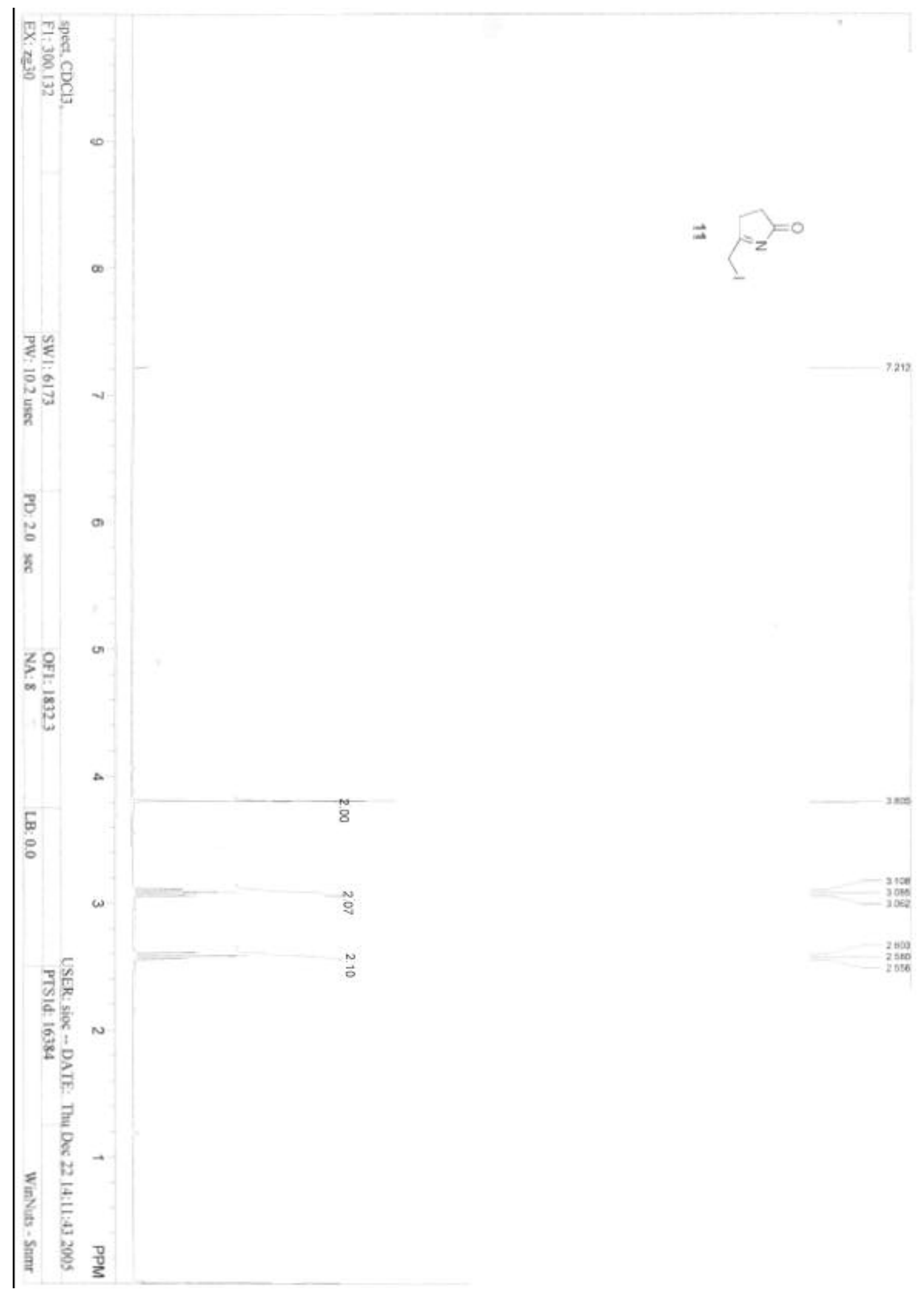




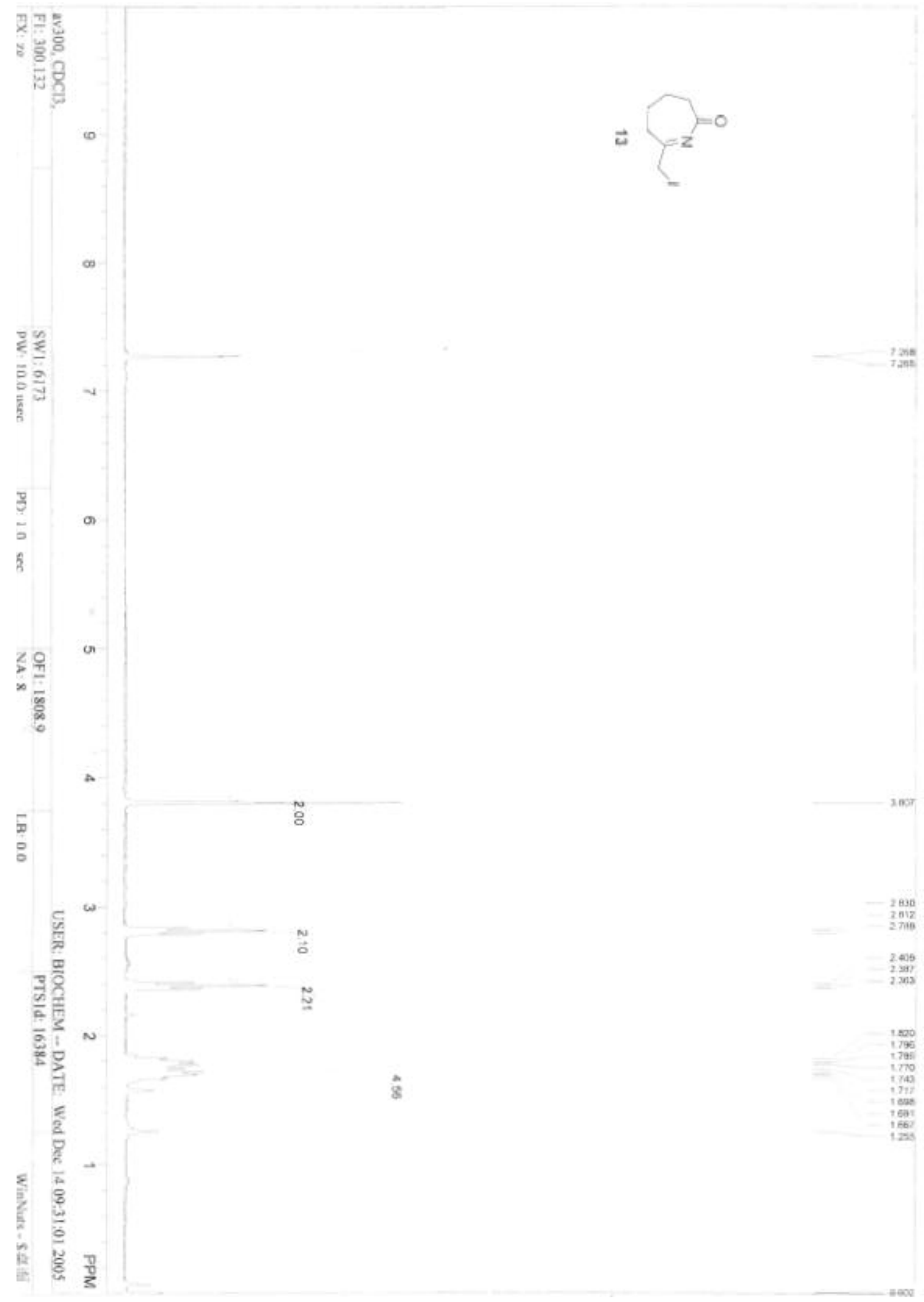




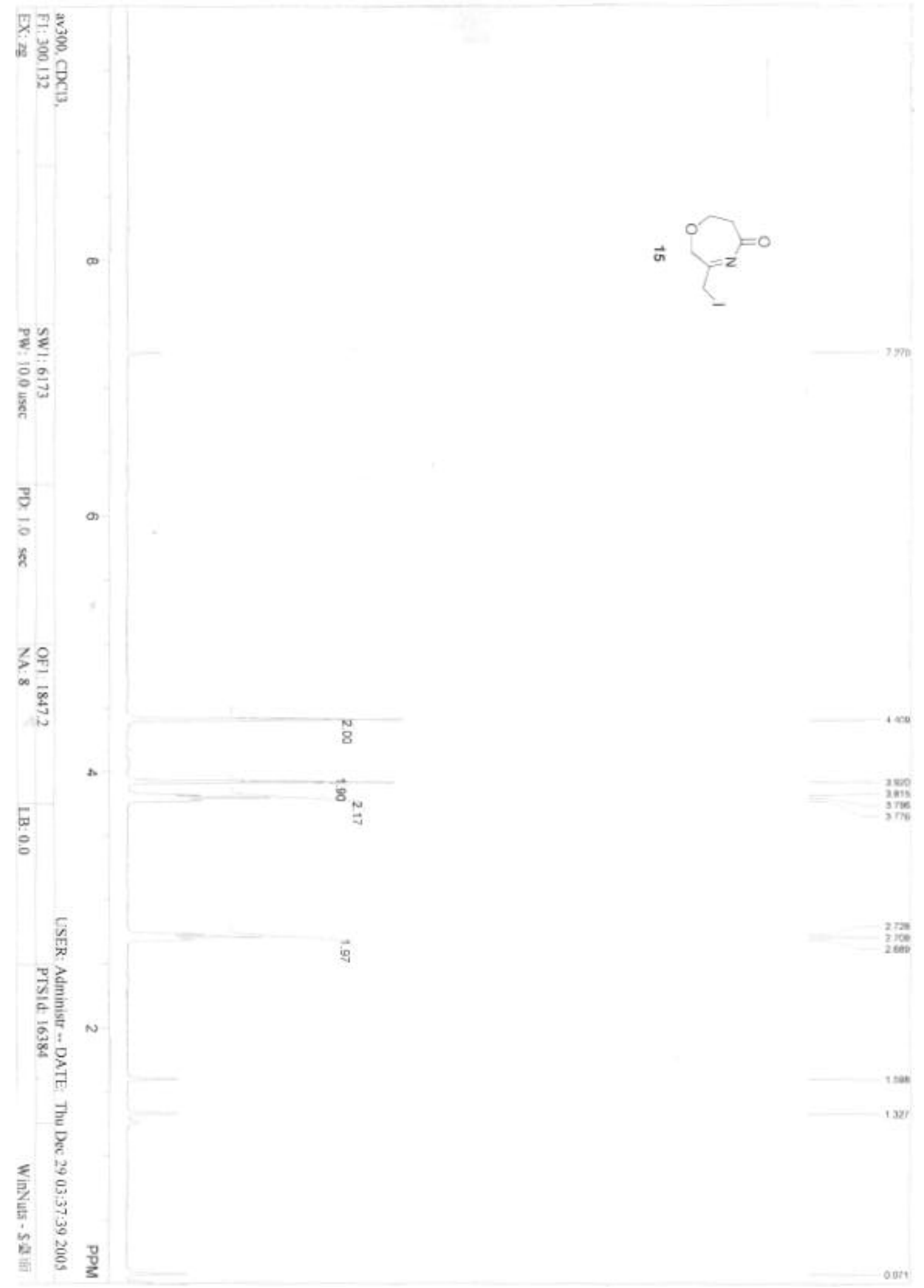




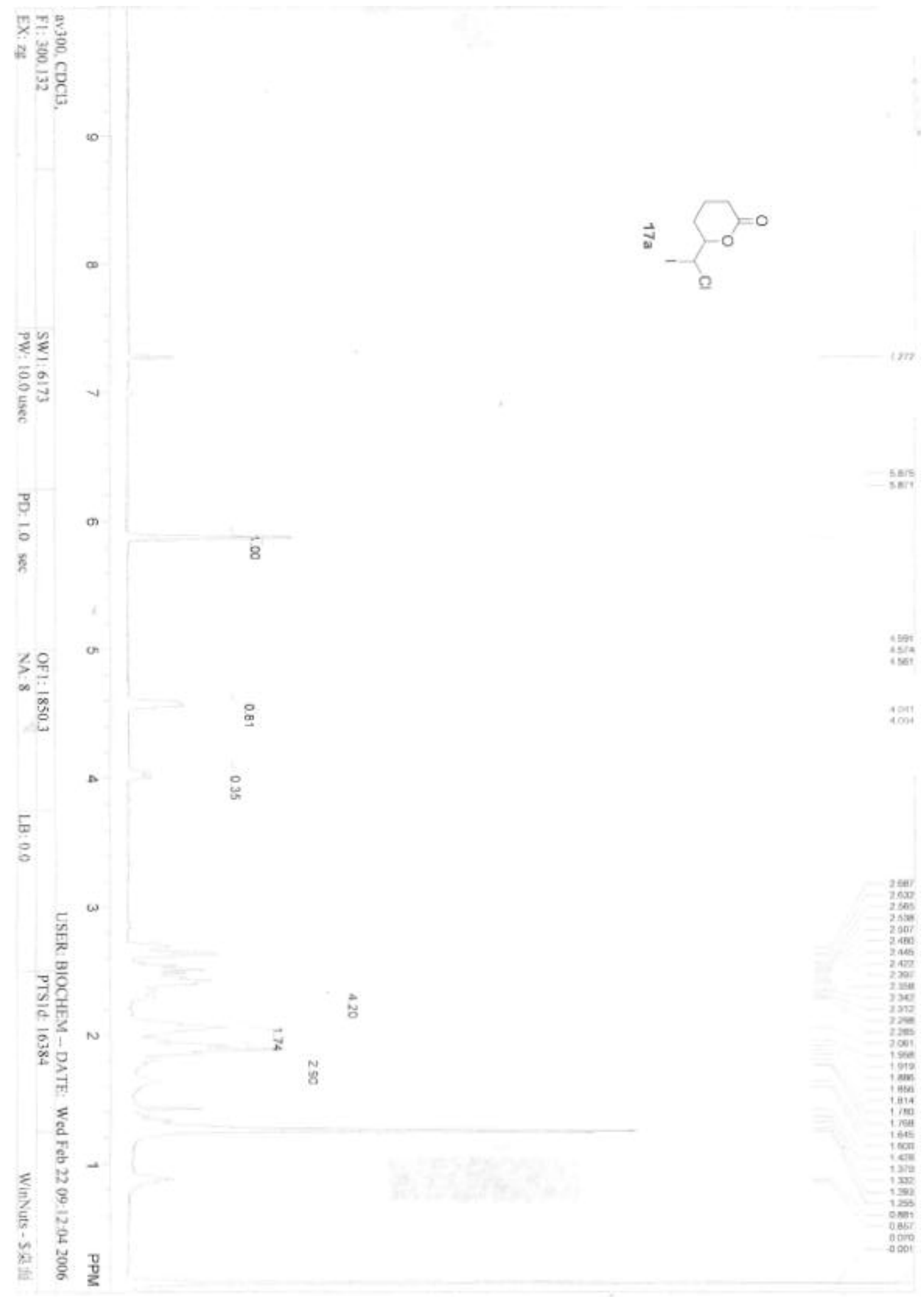



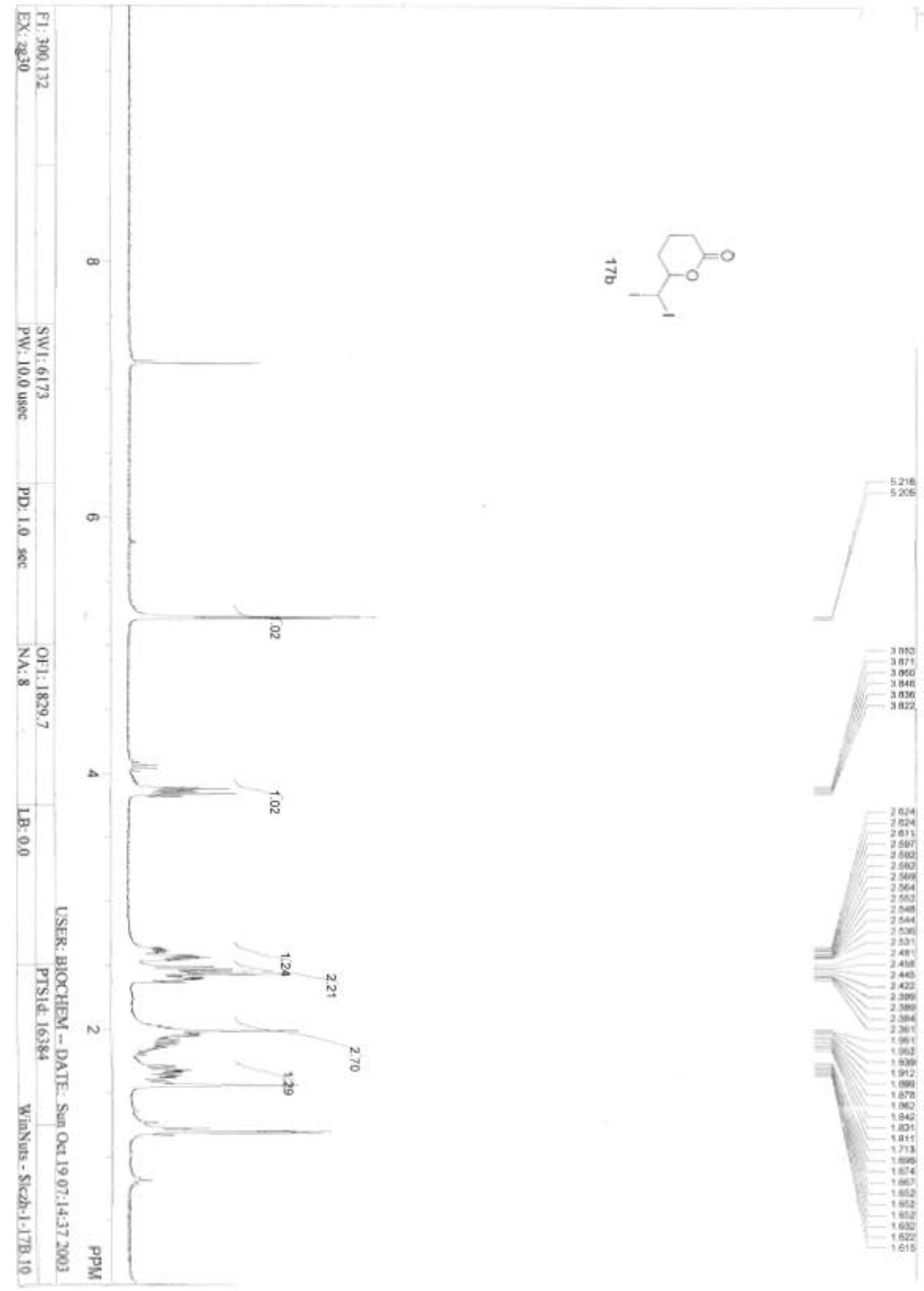


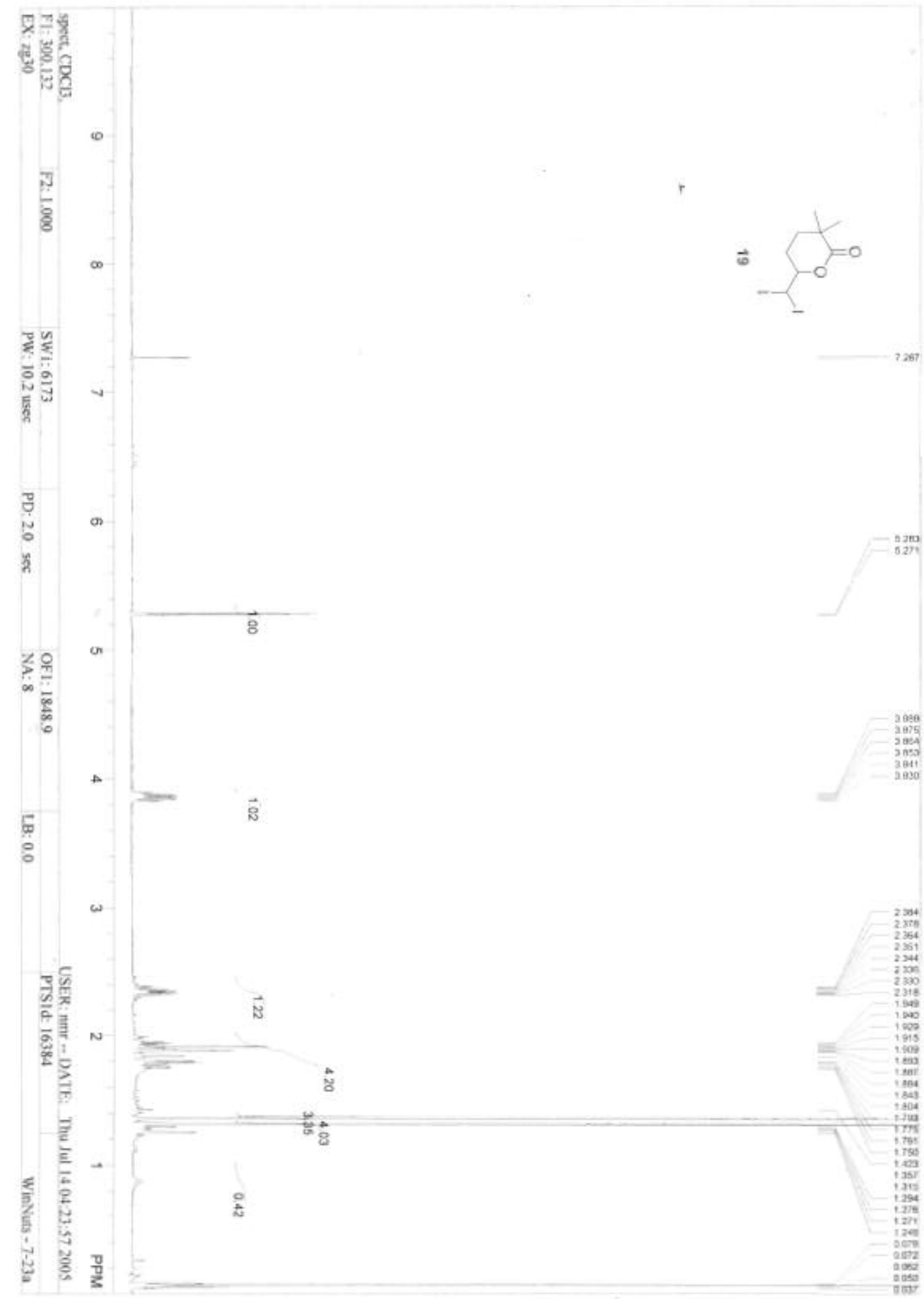




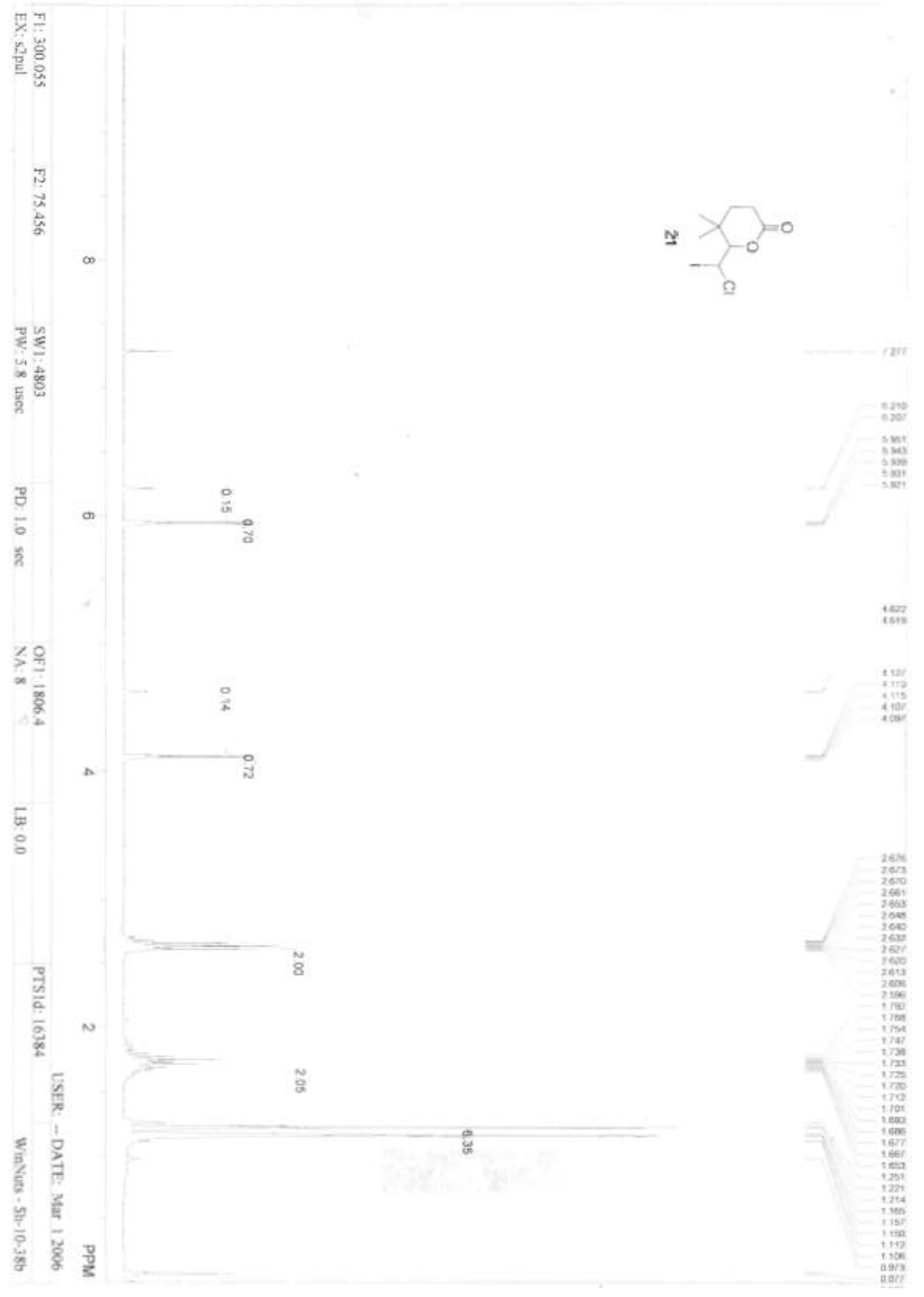




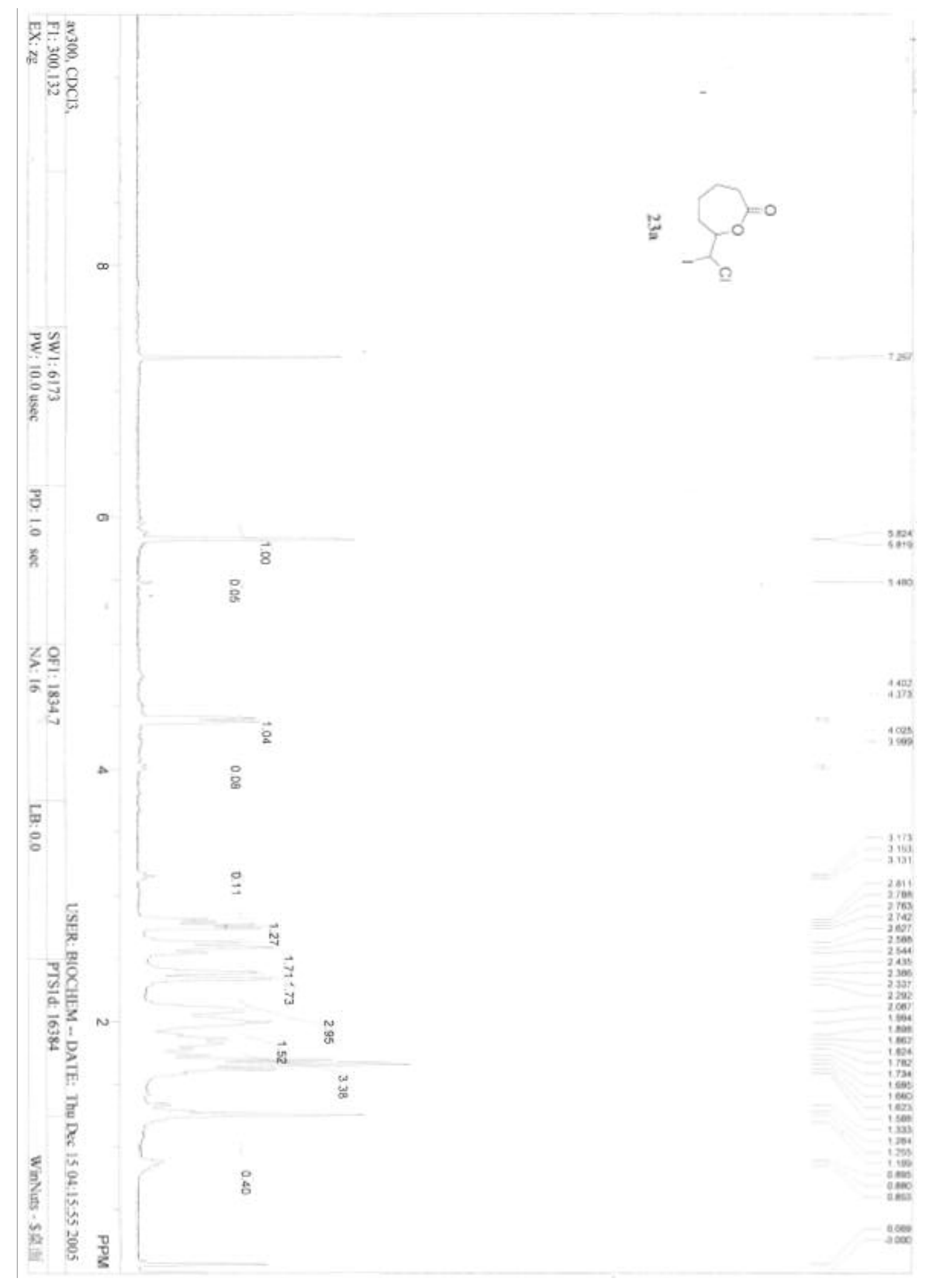




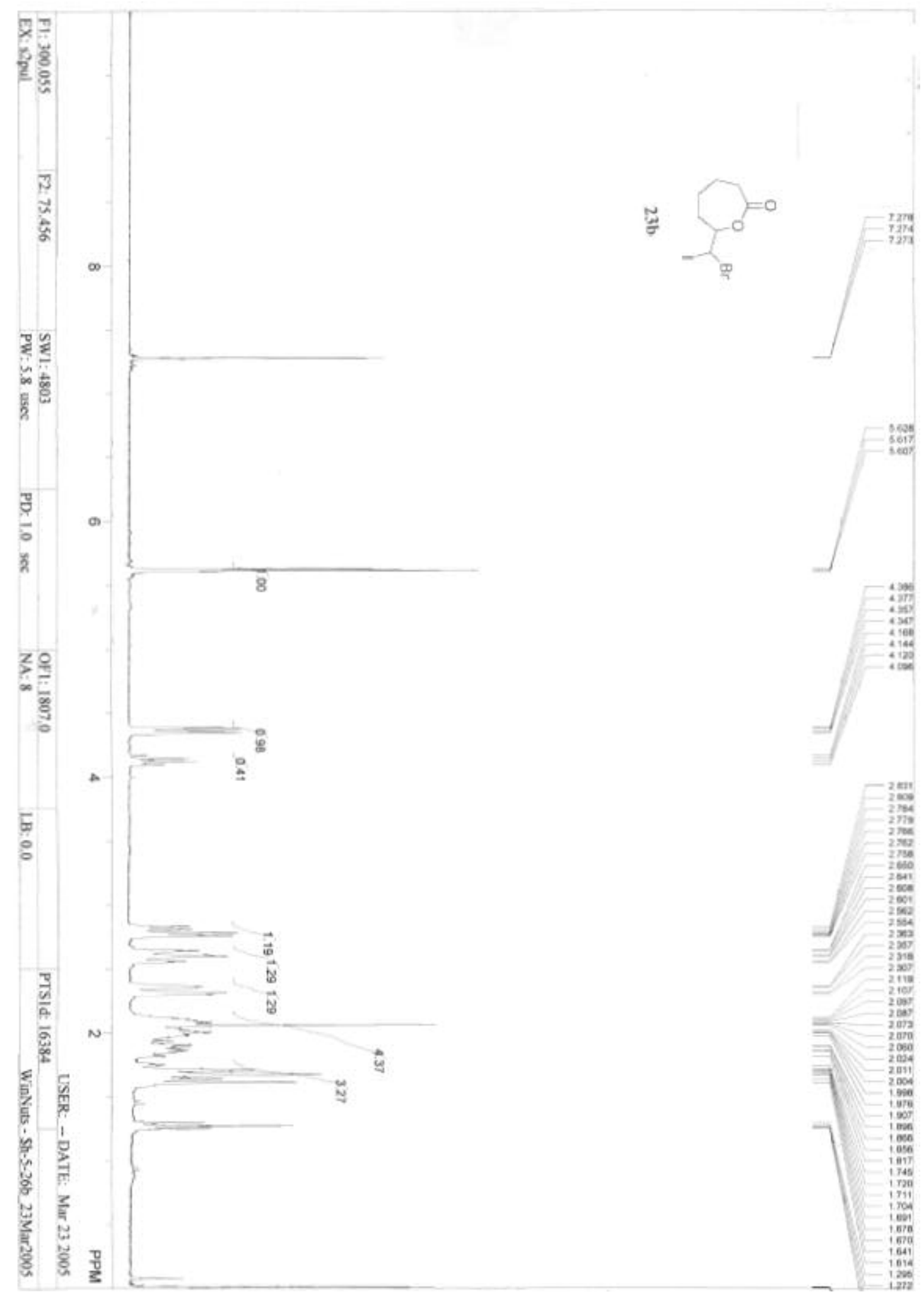




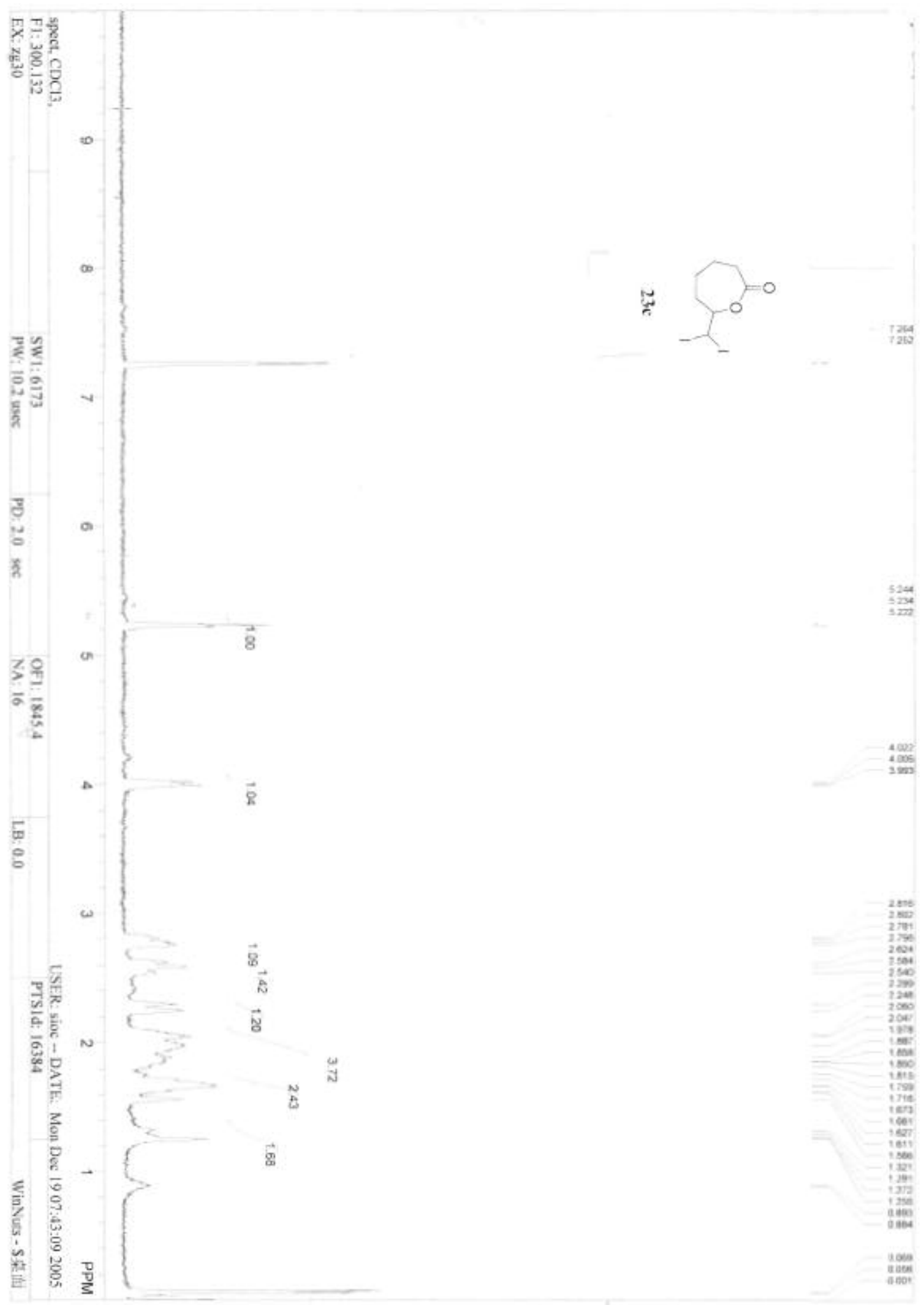

